\title{
On the theory of energy distributions of products of molecular beam reactions involving transient complexes*
}

\author{
R. A. Marcus \\ Department of Chemistry, University of Illinois, Urbana, Illinois 61801 \\ (Received 14 October 1974)

\begin{abstract}
Theoretical energy distributions of reaction products in molecular beam systems are described for reactions proceeding via transient complexes. Loose and tight transition states are considered for the exit channel. For a loose transition state and the case of $l>j$, the result is the same as of Safron et al. For the case of a tight transition state exit channel effects are included analogous to steric effects for the reverse reaction. It is shown how, via one mechanism, bending vibrational energy of that transition state can contribute to the translational energy of the reaction products. Expressions
\end{abstract} \\ are derived for the energy distributions of the products when $l>j$ and $j>l$.
}

\section{INTRODUCTION}

In recent years illuminating experiments on the behavior of long-lived collision complexes have been performed in molecular beam systems. ${ }^{1,2}$ The overall reaction can be represented as

$$
\begin{aligned}
& \mathrm{A}+\mathrm{BC}-\mathrm{ABC}^{*}, \\
& \mathrm{ABC} \mathrm{C}^{*}-\mathrm{ABC}^{\ddagger}-\mathrm{AB}+\mathrm{C},
\end{aligned}
$$

where $\mathrm{ABC}^{*}$ is the long-lived collision complex and $A B C^{\ddagger}$ is the transition state for the "exit" channel forming $\mathrm{AB}+\mathrm{C}$. [The transition state for $\mathrm{Eq}$. (1.1) has been omitted for brevity.] In these experiments the translational energy distribution of the reaction products was measured. In related experiments on some of these systems vibrational ${ }^{3}$ and rotational ${ }^{4}$ energy distributions of the reaction products have also been measured.

Comparison with the data has been made with an adaptation of RRKM theory for both "loose" and "tight" transition states, as well as with phase space theory. ${ }^{1-4}$ In chemical kinetics, loose transition state theory is appropriate for reactions not having steric factors, while tight transition state theory is needed for reactions having steric effects. ${ }^{5}$ Phase space theory ${ }^{6}$ is the same as transition state theory when the transition states for both (1.1) and (1.2) are loose and when angular momentum restrictions on the latter are fully taken into account (e.g., Sec. V).

It has been suggested that the molecular beam results can be used to test the energy randomization assumption of RRKM theory, ${ }^{7}$ although the latter theory was designed for calculating rates $s^{8,9}$ rather than for calculating energy distributions of reactions products. When the transition states are loose, no added assumptions are indeed needed: In a loose transition state $A B$ and $C$ rotate freely, and so their vibrational-rotational motion is uncoupled from the radial-orbital motions in $\mathrm{ABC}^{\ddagger}$ and $a$ fortior $i$ in the motion from $\mathrm{ABC}^{\ddagger}$ to the products $\mathrm{AB}+\mathrm{C}$. Thus, in this case RRKM theory can be used without further approximation for discussing energy distributions.

In the case of a tight transition state, only by adding additional assumptions to it can one adapt it to yield expressions for these distributions. Thus, only when the latter assumptions are valid do the beam data test RRKM theory itself.

In a recent stimulating theoretical study using transition state theory to obtain the energy distribution of reaction products, it was assumed that the distribution of vibrational and rotational energies in the products of (1.2) was the same as that in the transition state $A B C^{\ddagger} .{ }^{10}$ The translational energy distribution of relative motion of the products was assumed to be greater than that in $\mathrm{ABC}^{\ddagger}$ by a centrifugal plus potential energy term, and an appropriate distribution function for this term was included. The case of relatively large orbital angular momentum quantum numbers $l$ in (1.2) and large ones $l_{0}$ in $(1.1)$ was treated. ${ }^{10}$

Given the above energy distribution in $A B C^{\ddagger}$, what is of particular interest now is an examination of possible dynamical (or statistical and dynamical) effects in the evolution of the degrees of freedom of a tight $\mathrm{ABC}^{\ddagger}$ into those of $A B+C$. Such effects would further influence the internal and translational energy distributions of the reaction products, both for the case of large and small $l$. For example, some of the bending vibrational energy of $\mathrm{ABC}^{\ddagger}$ may be converted into translational energy of $A B+C$ in (1.2), as follows. ${ }^{11}$

When the transition state in (1.2) is tight, there are bending vibrations in $\mathrm{ABC}^{\ddagger}$ which tend to become rotations of the products. Because the spacing of bending vibration levels is wider than that of rotational levels, an extra energy is released into translational energy $E_{p}$ of the products if the conversion of the bending to rotational motion is "statistically adiabatic" (adiabatic on the average). This effect, when it occurs, would cause the translational energy of the products $\mathrm{AB}+\mathrm{C}$ to be shifted to higher values.

In the present paper an expression is derived for the energy distribution of the reaction products, taking this effect into account for a tight transition state. The relation between loose transition state theory and phase space theory is first summarized, so as to set a background for analysis of the tight transition state theory.

The organization of the paper is as follows. In Sec. II the transition states for some reactions are discussed, differentiating between loose and tight. In Sec. III the distribution of total angular momenta in (1.1) for a loose transition state for that reaction is given. In Sec. 
IV the assumptions used in treating (1.2) are listed. State-selected cross sections are derived for the case of a loose transition state for (1.2) in Sec. V and compared with those of phase space theory. Approximations are then given for the cases of $l \gg j$ and $j \gg l$, where $l$ and $j$ refer to the orbital and rotational angular momenta of the products of (1.2). The corresponding cases $\left(l \gg j\right.$ and $j \gg l$ ) when $\mathrm{ABC}^{l}$ is a tight transition state for (1.2) are treated in Sec. VI, and the former is illustrated with several examples in Sec. VII.

The distribution of translational energies of reaction products for the four limiting cases in Secs. V and VI is deduced in Sec. VIII from the state-selected product distributions given in those sections. Rotational and vibrational distributions are derived in Sec. IX. The paper concludes with some further comments in Sec. X.

\section{TRANSITION STATES FOR SOME REACTIONS}

When the reaction cross section for the forward step in (1.1) leading to the formation of an $\mathrm{ABC}^{*}$ is large, steric effects in that step are small and so the transition state of (1.1) can be regarded as loose. Because of detector sensitivity limitations the reactions studied have had relatively large cross sections and so the transition state has typically been regarded as loose for this first step.

The transition state for the second step, $A B C^{*}-A B$ $+C$, Eq. (1.2), however, is probably loose for some reactions but tight for others: Typically, one might expect that when the energy barrier for the reverse step $\mathrm{AB}+\mathrm{C} \rightarrow \mathrm{ABC}^{*}$ is large, the barrier will depend on the relative orientation of $A B$ and $C$, steric effects will therefore be present and the transition state will be tight. When the barrier is negligible for the more favorable directions of mutual approach, it might be nonnegligible for other directions, and some steric effects might still occur.

An example of a chemical activation whose exit channel (1.2) appears to involve no significant potential energy barrier for the reverse step, $\mathrm{AB}+\mathrm{C}-\mathrm{ABC}{ }^{*}$, is$$
\mathrm{F}+\mathrm{CRC} l=\mathrm{CR}^{\prime} \mathrm{R}^{\prime \prime}-\mathrm{FRC} l-\dot{\mathrm{C}} \mathrm{R}^{\prime} \mathrm{R}^{\prime \prime}-\mathrm{FCR}=\mathrm{CR}^{\prime} \mathrm{R}^{\prime \prime}+\mathrm{C} l,
$$

where the R's are alkyl or other groups. ${ }^{2,12 a}$ Treatment of such reactions by a loose transition state statistical theory in which all internal coordinates of $\mathrm{ABC}^{*}$ participated in sharing the excess energy yielded reasonable agreement with the data. ${ }^{2,7}$

Two other examples of chemical activation, the second of which has a large potential energy barrier ${ }^{12 b, 2}$ for the reverse step of $\mathrm{AB}+\mathrm{C} \rightarrow \mathrm{ABC}^{\ddagger}$, involve $\mathrm{H}$ atom ${ }^{2,7}$ or methyl radical ${ }^{2,7}$ elimination instead of $\mathrm{C} l$ elimination:

$$
\begin{aligned}
\mathrm{F}+\mathrm{CHR}= & \mathrm{CR}^{\prime} \mathrm{R}^{\prime \prime}-\mathrm{CHRF}-\dot{\mathrm{C}} \mathrm{R}^{\prime} \mathrm{R}^{\prime *}-\mathrm{FCR}=\mathrm{CR}^{\prime} \mathrm{R}^{\prime \prime}+\mathrm{H}, \\
\mathrm{F}+\mathrm{CH}_{3} \mathrm{CR} & =\mathrm{CR}^{\prime} \mathrm{R}^{\prime \prime}-\mathrm{CH}_{3} \mathrm{CRF}-\dot{\mathrm{C}} \mathrm{R}^{\prime} \mathrm{R}^{\prime *}-\mathrm{FCR} \\
& =\mathrm{CR}^{\prime} \mathrm{R}^{\prime \prime}+\mathrm{CH}_{3} .
\end{aligned}
$$

Thus, tight transition states would be expected for the dissociation step, at least in (2.3). On the basis of re- cent beam data, one could infer either that all vibrations of $\mathrm{ABC}^{*}$ do not participate in the energy sharing during the short life of the vibrationally hot molecule, ${ }^{2,7}$ or that the added assumptions used to calculate the energy distribution of $\mathrm{AB}+\mathrm{C}$ were in error, ${ }^{3}$ or possibly both., ${ }^{3,15}$ The added assumptions did not, for example, allow for any possible statistical-adiabatic effect described in the previous section. The effect is such that it would qualitatively reduce the discrepancy. Thus, numerical tests of the present or other models is needed for systems involving tight transition states, before more definite conclusions can be drawn regarding the number of vibrations of $\mathrm{ABC}^{*}$ participating in the energy sharing, where $\mathrm{ABC}^{\ddagger}$ is tight.

\section{DISTRIBUTION OF ANGULAR MOMENTA OF $A B C^{\ddagger}$}

We consider the case where the transition state of (1.1) is loose. The final results for this aspect of problem can be altered when this step has instead a tight transition state, by utilizing arguments analogous to those employed for (1.2) in Sec. VI. All previous work appears to have used a loose transition state for (1.1). ${ }^{1,2,7,10,14}$

Let $l_{0}$ and $j_{0}$ denote the orbital and the total rotational angular momentum quantum numbers for (1.1). The total angular momentum quantum number $\kappa$ lies in the interval $\left(\left|l_{0}-j_{0}\right|, l_{0}+j_{0}\right)$, by the usual rule for addition of angular momenta.

The loose transition state occurs at the maximum of the effective potential energy for the radial motion $r$ of $\mathrm{AB}$ and $\mathrm{C}$ : If $U_{0}(r)$ is the actual potential energy for motion from $r=\infty$ to an $r$ in the vicinity of the loose transition state, the effective potential for the radial motion $B_{l}^{0}(r)$ contains a centrifugal term

$$
B_{l}^{0}(r)=U_{0}(r)+\left[l_{0}\left(l_{0}+1\right) \hbar^{2} / 2 \mu_{0} r^{2}\right] .
$$

$\mu_{0}$ is the reduced mass of $\mathrm{A}$ and $\mathrm{BC}$. The transition state occurs at an $r, r_{l_{0}}^{\ddagger}$, where $B_{l}^{0}(r)$ has its maximum. ( $U_{0}$ contains a long-range attractive term.) That is,

$$
d B_{i}^{0}(r) / d r=0 \text { at } r=r_{i_{0}}^{\ddagger},
$$

and $d^{2} B_{i}^{0}(r) / d r^{2}$ is negative at $r_{i_{0}}^{\ddagger}$.

The reaction cross section for $\mathrm{A}+\mathrm{BC}$ to form initially an $\mathrm{ABC}^{*}$ in (1.1) is $\sigma_{0}$. The cross section is given by a standard relation ${ }^{15}$

$$
\sigma_{0}=\frac{\pi}{k_{0}^{2}} \sum_{i_{0}=0}^{\infty}\left(2 l_{0}+1\right) w_{l_{0} \nu_{0}}^{0},
$$

where $w_{l_{0} 0}^{0}$ is the reaction probability for a colliding pair $A$ and $B C$ having an initial orbital angular momentum quantum number $l_{0}$ and an initial relative velocity $v_{0} . \quad w^{0}$ is independent of the internal states of $\mathrm{A}$ and $\mathrm{BC}$, in the case of a loose transition state. $k_{0}$ is the wavenumber $p_{0} / \hbar\left(=\mu_{0} v_{0} / \hbar\right)$. $w^{0}$ is a step function of the energy excess $E_{p_{0}}-B_{l}^{0}\left(r_{i_{0}}^{t}\right)$, where $E_{p_{0}}$, the initial translational energy, is $\frac{1}{2} \mu_{0} v_{0}^{2}$ :

$$
\begin{aligned}
w_{l_{0} v_{0}}^{0} & =1 \text { if } E_{p_{0}}>B_{l}^{0}\left(r_{i_{0}}^{t}\right) \\
& =0 \text { if } E_{\rho_{0}}<B_{l}^{0}\left(r_{i_{0}}^{t}\right) .
\end{aligned}
$$


For a given $E_{p_{0}}$, therefore, $w^{0}$ is unity for all $l_{0}$ 's up to some maximum value $l_{o m}$, defined by

$$
B_{l}^{0}\left(r_{l_{0}}^{\ddagger}\right)<E_{p_{0}} \text { for } l_{0}<l_{o m} \text {. }
$$

Using (3.4) and (3.5) the sum in (3.3) now yields

$$
\sigma_{0}=\pi\left(l_{o m}+1\right)^{2} / k_{0}^{2} \text {. }
$$

Differences such as those between $l_{o m}$ and $l_{o m}+1$ will later be ignored.

The distribution of total angular momentum quantum number $\kappa$ in the $\mathrm{ABC}^{*}$ 's formed in (1.1) will be needed. In the case of a loose transition state for (1.1) the probability of obtaining a particular $\kappa$ for $\mathrm{ABC}^{*}$ depends only on a statistical factor: The probability of forming a particular $\kappa$ in step (1.1) equals the ratio of number of $\kappa$ states to total number of $j_{0}$ and $l_{0}$ states, namely, $(2 \kappa$ $+1) /\left(2 j_{0}+1\right)\left(2 l_{0}+1\right)$, where $\kappa$ lies in $\left(\left|j_{0}-l_{0}\right|, j_{0}+l_{0}\right)$. Thus, the probability that a pair $\mathrm{A}+\mathrm{BC}$ of a given $l_{0}$ and $v_{0}$ not only reacts but forms a state of given $\kappa$ is

$$
w_{l_{0} v_{0}}^{o, \kappa}=\frac{2 \kappa+1}{\left(2 j_{0}+1\right)\left(2 l_{0}+1\right)} w_{l_{0} v_{0}}^{0},\left(\left|j_{0}-l_{0}\right| \leqslant \kappa \leqslant j_{0}+l_{0}\right) .
$$

The distribution function of $\mathrm{ABC}^{*}$ 's formed in (1.1) with a given $\kappa, P_{0}(\kappa)$, is obtained by multiplying (3.7) by the weighting factor $2 l_{0}+1$, summing over all $l_{0}$ 's consistent with the given $\kappa$ and $j_{0}$, and dividing by a normalizing factor:

$$
P_{0}(\kappa)=\frac{\sum_{l_{0}=|\kappa-j 0|}^{k+j 0}\left(2 l_{0}+1\right) w_{l_{0,0}}^{o, k}}{\sum_{l_{0}=0}^{\infty}\left(2 l_{0}+1\right) w_{l_{0} v_{0}}^{0}} .
$$

Further, $w_{l_{0 v_{0}}}^{0}$ equals unity when $l_{0} \leqslant l_{o m}$ and zero when $l_{0}>l_{o m}$, according to (3.4) and (3.5). Also, $l_{0}$ cannot exceed $\kappa+j_{0}$. Thus, Eqs. (3.7), (3.8), and (3.3) yield

$$
P_{0}(\kappa)=\frac{\pi(2 \kappa+1)}{k_{0}^{2} \sigma_{0}\left(2 j_{0}+1\right)} \sum_{l_{0}=|\kappa-j|}^{l_{c}^{0}} 1 .
$$

The upper limit $l_{<}^{0}$ on the $l_{0}$ sum is either the energylimited value $l_{o m}$ defined by (3.5) or the angular momentum-limited value $\kappa+j_{0}$, whichever is the smaller. That is,

$$
l_{<}^{0}=\operatorname{smaller}\left\{l_{o m}, k+j_{0}\right\} .
$$

The maximum value of $\kappa, \kappa_{m}$, for the given $j_{0}$, is the maximum value of $l_{0}+j_{0}$, i.e.,

$$
\kappa_{m}=l_{o m}+j_{0} .
$$

One may verify by interchanging the order of summations over $\kappa$ and $l_{0}$ that $^{16}$

$$
\sum_{k=0}^{\kappa=\kappa_{m}} P_{0}(\kappa)=1
$$

as indeed it should. The limits in the reordered sums are given below by (3.13) since, for a given $j_{0}$ and $l_{0}, \kappa$ lies in the interval $\left(\left|j_{0}-l_{0}\right|, j_{0}+l_{0}\right)$, and since $l_{0}$ itself must lie in the interval $\left(0, l_{a m}\right)$ :

$$
\left|j_{0}-l_{0}\right| \leqslant \kappa \leqslant j_{0}+l_{0}, \quad 0 \leqslant l_{0} \leqslant l_{\text {om }} \text {. }
$$

A useful simplification of (3.9) arises, one which will not be used until Sec. VB, when $l_{0} \gg j_{0}$. In this case, since the $l_{0}$ 's are clustered around $\kappa$, we may set in (3.9) and (3.10) (a) $\left|\kappa-j_{0}\right|=\kappa-j_{0}$ and (b) $l_{<}^{0}=\kappa+j_{0}$ for the large majority of $\kappa$ 's. The sum over $l_{0}$ in (3.9) then becomes $2 j_{0}+1$. Further, we may set $P_{0}(\kappa)$ equal to zero for $\kappa$ greater than $l_{o m}$, since $\kappa$ cannot exceed $l_{o m}+j_{0}$, and this quantity is essentially $l_{o m}$. Thus, Eqs. (3.9) and (3.6) yield

$$
\begin{aligned}
P_{0}(\kappa) & =(2 \kappa+1) / l_{o m}^{2}, & & \kappa<l_{o m} \\
& =0, & & \kappa>l_{o m}
\end{aligned}
$$

ignoring the difference in $l_{o m}^{2}$ and $\left(l_{o m}+1\right)^{2}$, for notational brevity.

\section{ASSUMPTIONS}

It will be assumed that noted,

(i) the transition state for (1.1) is loose, as already

(ii) $s$ vibrations of $\mathrm{ABC}^{*}$ participate in the energy sharing process, i.e., are active (the remaining, if any, are adiabatic throughout (1.1) and $\left.(1.2)^{17}\right)$;

(iii) transition state theory is valid for (1.2);

(iv) in the case of a tight transition state, an additional assumption given later.

\section{Assumption (iii) can be rephrased as}

(iii) a quasiequilibrium approximation for (1.2) ("microcanonical activated complex theory"), ${ }^{18}$ namely, Eq. (4.3) below, is valid.

Assumption (i) can be replaced, as noted in the previous section; the transition state for (i) could be tight and other reaction probabilities $w_{l_{0} v_{0}}^{o \kappa}$ could be introduced.

When the transition state $\mathrm{ABC}^{\ddagger}$ for $\mathrm{Eq}$. (1.2), is loose the assumptions (i)-(iii) permit an immediate calculation of the energy distribution of the reaction products. The results are given in Sec. V, and are the same as those of phase space theory, as already noted. They reduce to those of a recently formulated loose transition state theory ${ }^{10}$ for the reaction (1.2) when one introduces the approximations, $\kappa \cong l_{0}$ and $l \cong \kappa, l$ being the orbital angular momentum quantum number of the products. That transition state theory employed these useful approximations, and so this last result, too, is the expected one. Another limiting case, $l_{0} \cong \kappa \cong j$, is also given in Sec. $V$.

There may be several sets of reaction products from $\mathrm{ABC}^{*}$ in reaction (1.1)-(1.2), besides $\mathrm{AB}+\mathrm{C}$ and $\mathrm{A}+\mathrm{BC}$. We denote by $\alpha$ the $\alpha$ th set of products, and introduce the following additional symbols for reaction probabilities and cross sections: $\sigma_{j n_{j} j_{0} n_{0} E}^{\alpha}$ is the cross section for forming the $\alpha$ th set of products in a rotational-vibrational state $j n$ from reactants in a state $j_{0} n_{0}$ when the total energy is $E ; \sigma_{\bar{B}_{p} ; j_{0} m_{0} E}^{\alpha} d E_{p}$ is the cross section for forming the $\alpha$ th set of products with a translational energy $\left(E_{p}, E_{p}+d E_{p}\right) ; w_{j n l B}^{\alpha \alpha}$ is the probability of forming an ABC* from a collision of the $\alpha$ th set of products having a given $j, n, l, \kappa$, and $E$.

The second $\sigma$ is related to the first by 
$\sigma_{E_{p} ; j_{0}^{n} 0^{E}}^{\alpha} d E_{p}=\sum_{j n} \sigma_{j n ; j 0^{n} 0^{B}}^{\alpha},\left(E-E_{p}-d E_{p} \leqslant E_{j n}^{\alpha} \leqslant E-E_{p}\right)$,

where the sum is such that the rotational-vibrational energy $E_{j n}^{\alpha}$ of the $\alpha$ th set of products satisfies the condition in parentheses.

Throughout, $j_{0}$ and $j$ denote the total rotational angular momentum of the reactants and of the $\alpha$ th set of products, respectively. The remaining rotational quantum numbers and the vibrational quantum numbers are included in $n_{0}$ and $n$, which are discussed more fully in Sec. IX.

Assumption (iii) for a given $\kappa$ and $E$ can be written as $^{19}$

$\sum_{j n l} w_{j n l E}^{\kappa \alpha}=\sum_{n \ddagger} 1 \equiv N_{\kappa \alpha}^{\ddagger}, \quad\left(|\kappa-j| \leqslant l \leqslant \kappa+j, 0 \leqslant E_{j n}^{\alpha} \leqslant E\right)$,

where the first sum is over all $j, n$, and $l$ consistent with the given $\kappa, E$, and $\alpha$, namely, over $j, n$, and $l$ satisfying the conditions in parentheses. The sum over $n^{t}$ in (4.2) is over all quantum states of the $\mathrm{ABC}^{t}$ for the $\alpha$ th set of products, consistent with the given $E$ and $\kappa$. $N_{\kappa \alpha}^{4}$ denotes the number of such states, apart from a degeneracy factor $2 \kappa+1$, which is absent from both sides of (4.2).

To simplify the notation the subscript $\alpha$ present above will be omitted in the future from all symbols, apart from $N_{\text {k } \alpha}^{\ddagger}$.

The total cross section for producing the $\alpha$ th set of products, $\sigma_{j_{0} n_{0} E}$, for the given $j_{0}, n_{0}$, and $E$ is, in the present statistical-type theory,

$$
\sigma_{j 0^{n_{0} E}}=\sigma_{0} \sum_{\kappa=0}^{\kappa_{m}} P_{0}(\kappa) \frac{N_{k \alpha}^{t}}{\sum_{\alpha} N_{k \alpha}^{\ddagger}},
$$

since $N_{k \alpha}^{\ddagger} / \sum_{\alpha} N_{k \alpha}^{\ddagger}$ is the probability that a transition state of a given $\kappa$ will be of the $\alpha$ th type, and $\sigma_{0} P_{0}(\kappa)$ is the contribution of a given $\kappa$ to $\sigma_{0}$.

When (4.2) is introduced for $N_{k \alpha}^{\ddagger}$ into the numerator of (4.3), we have

$$
\begin{aligned}
\sigma_{j_{0} n_{0} E} & \equiv \sum_{j n} \sigma_{j n, j} 0_{00^{E}} \\
& =\sigma_{0} \sum_{\kappa=0}^{\kappa} \frac{P_{0}(\kappa)}{\sum_{\alpha} N_{\kappa \alpha}^{\ddagger}} \sum_{j n l} w_{j n t E}^{\kappa},
\end{aligned}
$$

where the conditions in (4.2) on the $j n l$ sum apply. The sums on the right-hand side can be reordered, without any change in limits, to read $\sum_{f_{n}} \sum_{x} \sum_{l}$.

The right-hand side of (4.4) is now seen to be the sum of disjoint terms, since the reaction probability for $\mathrm{AB}$ $+\mathrm{C}-\mathrm{ABC}^{*}$ starting from any $\kappa j n$ state of $\mathrm{AB}+\mathrm{C}, \sum_{l} w_{j n t B}^{k}$, is independent of that starting from another $\kappa j n$ state at the same $E$ and $\alpha$. Thus, terms of the same $j$ and $n$ on both sides of the second half of (4.4) may be equated, yielding

$$
\sigma_{j n ; j_{0} 0_{0} E}=\sigma_{0} \sum_{\kappa=0}^{\kappa_{m}} \frac{P_{0}(\kappa)}{\sum_{\alpha} N_{\kappa \alpha \alpha}^{\ddagger}} \sum_{l=1 \kappa-j \mid}^{\kappa+\xi} w_{j n l E}^{\kappa},
$$

where $\kappa_{m}$ is given by $(3.11)$.

Assumption (iv) will be taken to be: $w_{j n t E}^{k}$ is a function of the energy excess for overcoming the barrier for the reverse of (1.2), $\mathrm{AB}+\mathrm{C} \rightarrow \mathrm{ABC}^{*}$. There is some minimum barrier, denoted by $B_{l}^{\ddagger}$, in addition to the extra component, present statistically, from the conversion of rotations of $\mathrm{AB}+\mathrm{C}$ into bending vibrations of a tight $A B C^{\ddagger}$. The energy excess is taken to be $E_{p}-B_{l}^{\ddagger}$. Thus,

$$
w_{j n l E}^{\kappa}=w^{\kappa}\left(E_{p}-B_{l}^{\ddagger}\right) \text {. }
$$

By using microcanonical activated complex theory [assumption (iii)] one introduces an effect arising from the difference of densities of states of bending vibrations in a tight $\mathrm{ABC}^{\ddagger}$ and of rotations in $\mathrm{AB}+\mathrm{C}$, due to the difference in energy spacing of quantum states. Assumption (iv) permits the translational energy to help overcome this mean difference in bending vibrational and rotational energy.

The simplest value for $B_{l}^{\ddagger}$ would be

$$
B_{l}^{\ddagger}=U^{\ddagger}+E_{l}^{\ddagger} \text {, }
$$

where $U^{\ddagger}$ and $E_{l}^{\ddagger}$ denote the potential energy of the tight transition state $\mathrm{ABC}^{\ddagger}$, relative to the $\alpha$ th set of products, and a centrifugal-type barrier, respectively:

$$
E_{l}^{\ddagger}=l(l+1) \hbar^{2} / 2 I^{\ddagger} \text {. }
$$

Here, $I^{\ddagger}$ is a relevant moment of inertia of ABC. The assumption of $U^{t}+E_{l}^{\ddagger}$ as the minimal barrier provides a simple way for assuring that appropriate impact parameters for the reverse step in (1.2) occur, via a suitable $I^{\ddagger}$. More complex expressions for $B_{l}^{\ddagger}$ could be tried instead. Some of the above symbols are illustrated in Fig. 1.

Equations (4.6)-(4.8) automatically apply to a loose transition state, with $I^{\ddagger}$ replaced by $\mu R_{l}^{\ddagger 2}$, where $R_{i}^{\ddagger}$ is defined in Sec. V, and with $w^{\kappa}$ being the simple step function given in Sec. V by Eq. (5.4). For a tight transition state the moments of inertia could depend slightly on the quantum numbers, but usually in chemical kinetics any such dependence is ignored when the transition state is tight.

The use of several additional symbols will prove helpful. We denote by $l_{m}$ the upper limit of the $l$ 's satis-

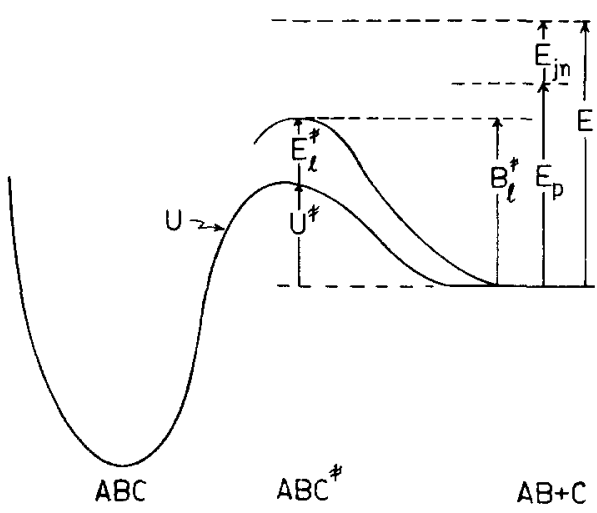

FIG. 1. Properties in the exit channel. $U \ddagger$ may be positive, as indicated, or negative. 


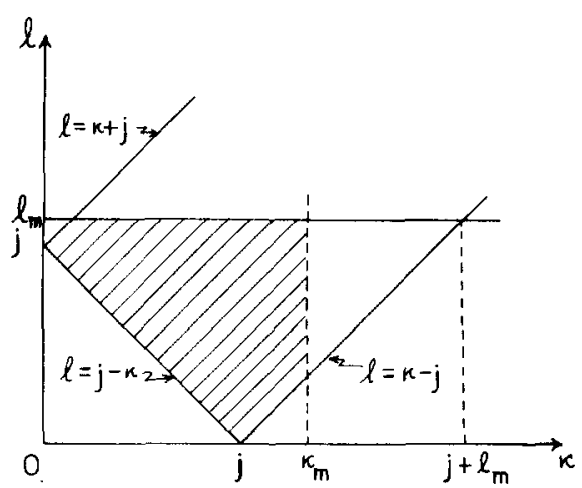

FIG. 2. Plot of $(l \cdot k)$ quantum states's space available (shaded area) for forming products of a given $j$ and $E_{p}$. The line $\kappa=\kappa_{m}$ is drawn for the case that $j+l_{m} \geq \kappa_{m}>j$.

fying the condition $B_{l}^{\ddagger}<E_{p}$. That is,

$$
B_{i}<E_{p} \text { for } l<l_{m}\left(E_{p}\right) \text {. }
$$

$l_{m}$ thus depends only on $E_{p}$.

Since $l$ can exceed neither $l_{m}$ nor $\kappa+j$ and since $\kappa$ can exceed neither $\kappa_{m}$ nor $l_{m}+j$, Eq. (4.5) can be rewritten as

$$
\sigma_{j n ; j_{0} n_{0} E}=\sigma_{0} \sum_{\kappa=0}^{\kappa} \frac{P_{0}(\kappa)}{\sum_{\alpha} N_{\kappa \alpha}^{\ddagger}} \sum_{l=\{\kappa=j \mid}^{i_{<}} w_{j n l E}^{\kappa},
$$

where

$$
\begin{aligned}
& l_{<}=\text {smaller }\left\{j+\kappa, l_{m}\right\}, \\
& \kappa_{<}=\operatorname{smaller}\left\{j+l_{m}, \kappa_{m}\right\},
\end{aligned}
$$

and $\kappa_{m}$ is given by (3.11). Thus, the upper limit of $l$ is either an angular-momentum-limited value $j+\kappa$ or an energy-limited value $l_{m}$. Similarly, the upper limit of $\kappa$ is either $j+l_{m}$ or $\kappa_{m}$, (both are energy-limited plus angular-momentum-limited values).

In two of the cases (Secs. V C and VIC) it will be convenient to interchange the order of the sums in (4.10). The new limits are seen from Fig. 2 to be

$$
\sigma_{j n ; j_{0} n_{0} E}=\sigma_{0} \sum_{l=0}^{i_{m}} \sum_{\kappa=|j,-l|}^{\kappa_{\varepsilon}^{\prime}} \frac{P_{0}(\kappa)}{\sum_{\alpha} N_{k \alpha}^{t}} w_{j n l E}^{\kappa},
$$

where

$$
\kappa_{<}^{\prime}=\operatorname{smaller}\left\{j+l, \kappa_{m}\right\} .
$$

In Sec. VI we shall need the following densities of states of the $\alpha$ th set of products.

The number of active vibrational-rotational $j n$ states of the $\alpha$ th products when their energy $E_{3 n}$ lies in the interval $\left(E-E_{p}-d E_{p}, E-E_{p}\right)$ will be denoted by $\rho\left(E-E_{p}\right)$ $\times d E_{p}$ and equals

$\rho\left(E-E_{p}\right) d E_{p} \equiv \sum_{j n}(2 j+1),\left(E-E_{p}-d E_{p} \leqslant E_{j n} \leqslant E-E_{p}\right)$.

Depending on the approximation used for $\rho$, $\rho$ can consist wholly of a sum of delta functions, or of delta functions (for the vibrational eigenvalues) superimposed on a continuous function, or of a continuous function.

A second density of states needed in Sec. VI involves the sum of all $n$ states in an energy range for systems with a given $j$ :

$$
\rho^{j}\left(E-E_{p}\right) d E_{p}=\sum_{n} 1,\left(E-E_{p}-d E_{p} \leqslant E_{j n} \leqslant E-E_{p}\right) .
$$

\section{LOOSE TRANSITION STATE FOR ABC ${ }^{\ddagger}$}

\section{A. Loose transition state for any $\ell$ and $j$}

If $U(R)$ denotes the potential energy for the radial motion $R$ in (1.2) in the vicinity of the transition state and for larger $R^{\prime} \mathrm{s}$, the effective potential for the $R$ motion $B_{l}(R)$ is

$$
B_{l}(R)=U(R)+l(l+1) \hbar^{2} / 2 \mu R^{2} .
$$

The transition state occurs at an $R=R_{l}^{\ddagger}$ where $B_{l}(R)$ has its maximum. $R_{l}^{\ddagger}$ depends only on $l$, the orbital angular momentum quantum number of the products $\mathrm{AB}+\mathrm{C}$. The value of $B_{l}$ at $R=R_{l}^{\ddagger}$ is denoted by $B_{l}^{\ddagger}$.

The quantum numbers for $\mathrm{ABC}^{\ddagger}$ are $\kappa, j, l$, and $n$ when $\mathrm{ABC}^{\ddagger}$ is a loose transition state, and all states of this loose $\mathrm{ABC}^{\ddagger}$ are equally probable a priori, for the given $\kappa$ and $E$. The total number of such states available is $\sum_{\alpha} N_{\kappa \alpha}^{t}$, where

$$
N_{\kappa \alpha}^{t}=\sum_{j} \sum_{n} \sum_{l=|k-j|}^{t_{<}} 1,\left(0 \leqslant E_{j n} \leqslant E\right),
$$

and $l_{<}$is given by (4.11).

Since $j, n$, and $l$ are good quantum numbers along the reaction coordinate $R$ from $\mathrm{ABC}^{t}$ to $\mathrm{AB}+\mathrm{C}$, the distribution of $j$ and $n$ in $\mathrm{AB}+\mathrm{C}$ is the same as that in $\mathrm{ABC}$. All states of $\mathrm{ABC}^{\ddagger}$ contributing to (5.2) are equally probable. Thus, the probability of finding a transition state in any one of these states is $1 / \Sigma_{\alpha} N_{k \alpha}^{t}$, for the given $\kappa$ and $E$. Since the probability of finding an $\mathrm{ABC}^{*}$ with any given $\kappa$ is $P_{0}(\kappa)$, and since the total cross section for forming an $\mathrm{ABC}^{*}$ in (1.1) is $\sigma_{0}$, the cross section for forming any $j$ and $n$ is obtained by summing over all $l$ consistent with this $j, \kappa$, and $n$, and then summing over $\kappa$ :

$$
\sigma_{i n ; j_{0} n_{0} E}=\sigma_{0} \sum_{\kappa=0}^{\kappa<} P_{0}(\kappa) \sum_{i=|\kappa-j|}^{i_{<}}\left(1 / \sum_{\alpha} N_{\kappa \alpha}^{ \pm}\right),
$$

where $l_{<}$and $\kappa_{<}$are given by (4.11) and (4.12).

Equations (5.3) and (3.9) are equivalent to the phase space theory result. ${ }^{20}$

For later comparison we note parenthetically that the reaction probabilities $w_{j n l E}^{k}$ for the reverse of (1.2), defined in Sec. IV, is given by the following equation, in the case of a loose transition state:

$$
\begin{aligned}
w_{\text {JnlE }}^{k} & =1,\left(E_{p}>B_{l}^{\mathfrak{q}}\right) \\
& =0,\left(E_{p}<B_{l}^{\ddagger}\right) .
\end{aligned}
$$

Equation (5.3) could also have been obtained from (4.10) using (5.4).

\section{B. Loose transition state when $\ell_{0} \gg j_{0}$ and $\ell \gg j$}

A useful simplification of (5.3) arises when $l_{0} \gg j_{0}$ and $l \gg j$ for the more important $l_{0}$ 's and $l$ 's contributing to 


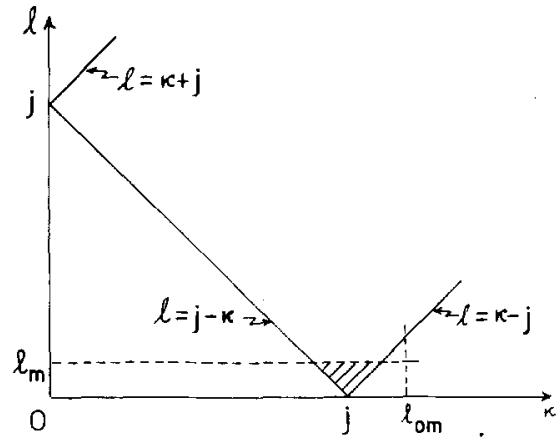

FIG. 3. Plot of $(\mathfrak{l}, \kappa)$ quantum states's space available (shaded area) for forming products of a given $j$ and $E_{p}$ for the case of $j \gg l$. The line $\kappa=\kappa_{m}$ is drawn for the case that $\kappa_{m}>j+l_{m}$.

(5. 3). It has already been seen that when $l_{0} \gg j_{0}$, Eq. (3.9) for $P_{0}(k)$ reduces to (3.14).

When $l \gg j$ the values of $l$ in (5.3) are largely clustered around the given value of $\kappa$ being considered. Thereby, in the sum over $l$ in (5.3), since $j$ is so small, we can set (a) $l_{<}=\kappa+j$, for the large majority of the $\kappa^{\prime} s$, and (b) $|\kappa-j|=\kappa-j$. Thus, the sum over $l$ in (5.3) equals $2 j+1$.

The upper limit $\kappa<$ in the sum over $\kappa$ in (5.3) is seen from (4.12) to be the smaller of the $l_{m}$ and $l_{o m}$, since $j+l_{m} \cong l_{m}$ and $\kappa_{m} \cong l_{o m}$. Equations (5.3) and (3.14) now yield the following equation, since the sum of $2 \kappa+1$ from $\kappa=0$ to $\kappa_{m}$ is $\kappa_{m}^{2}$ (neglecting unity relative to $\kappa_{m}$ ):

$$
\sigma_{j n ; j_{0} n^{B} E}=\frac{(2 j+1) \sigma_{0}}{\sum_{\alpha} N_{k \alpha}^{\ddagger}} A\left(E_{p}\right),
$$

where

$$
\begin{aligned}
A\left(E_{p}\right) & =1,\left(l_{m} \geqslant l_{o m}\right) \\
& =\left(l_{m} / l_{o m}\right)^{2},\left(l_{m} \leqslant l_{o m}\right) .
\end{aligned}
$$

[If unity had not been neglected relative to $l_{m}$ and to $l_{o m}$, the $\left(l_{m} / l_{o m}\right)^{2}$ in $(5.6 \mathrm{~b})$ would have been $\left(l_{m}+1\right)^{2} /\left(l_{o m}+1\right)^{2}$. The same remark applies later in (5.8b).] We have replaced the $\kappa$ in $\sum_{\alpha} N_{\kappa \alpha}^{t}$ by some mean value, an approximation which is a convenience rather than a necessity, and have introduced a notation $A\left(E_{p}\right)$ to facilitate comparison with Ref. $10^{21}$

\section{Loose transition state when $\ell_{0} \gg j_{0}$ and $j \gg \ell$}

It will be convenient to use (4.13) for $\sigma_{j n \text { j } 0 \pi_{0} B}$, with $w_{j n l E}^{x}$ being replaced by unity for this case of a loose transition state [since $l \leqslant l_{m}$ in (4.13)]. The $\kappa$ in $\sum_{\alpha} N_{k \alpha}^{t}$ in (4.13) will be replaced by some averaged value, and the $P_{0}(\kappa)$ in (4.13) is given by (3.14).

Figure 3 shows that in evaluating the sums over $\kappa$ and $l$ in (4.13) it is convenient to consider four cases which differ in the value of $\kappa_{m}:$ (1) $\kappa_{m} \geqslant j+l_{m}$, (2) $j+l_{m} \geqslant \kappa_{m}>j$, (3) $j \geqslant \kappa_{m}>j-l_{m}$, and (4) $j-l_{m}>\kappa_{m}$. In the present case $j \gg l_{m}$ and so in practice one need consider mainly cases (1) and (4), with (2) and (3) only providing some small transition region.

For the case of $\kappa_{m} \geqslant j+l_{m}$ the $\kappa_{<}^{\prime}$ in (4.14) becomes $j+l$, the $|j-l|$ in (4.13) is $j-l$ (since $j>l$ ), and so in (4.13) the sum over $\kappa$ becomes a sum of $2 \kappa+1$ from $j-l$ to $j+l$. The value of this sum is $(2 j+1)(2 l+1)$. The sum of $2 l+1$ from 0 to $l_{m}$ then yields $l_{m}^{2}$ (neglecting unity relative to $\left.l_{m}\right)$.

Considering next the case $\kappa_{m}<j-l_{m}$, we note that $\kappa$ cannot simultaneously satisfy the angular momentum addition rule, $j-l_{m} \leqslant \kappa \leqslant j+l_{m}$, and the condition $\kappa \leqslant \kappa_{m}$ $<j-l_{m}$. Thus, the corresponding $\sigma_{j n ; j_{0} n_{0} E}$ vanishes.

These two cases can be written as $l_{o m}>j$ and $l_{o m}<j$, noting that $\kappa_{m} \cong l_{o m}$ and $l_{m} \ll j$. Equations (4.13), (5.4), and (3.14) thus yield

$$
\sigma_{j n ; j_{0} n_{0} E} \cong \frac{(2 j+1) \sigma_{0}}{\sum_{\alpha} N_{k \alpha}^{!}} A^{\prime}\left(E_{p}\right)
$$

where

$$
\begin{aligned}
A^{\prime}\left(E_{p}\right) & =0 & & \text { for } j>l_{o m} \\
& =\left(l_{m} / l_{o m}\right)^{2}, & & \text { for } j<l_{o m} .
\end{aligned}
$$

Equation (5.7) plus (5.8b) is identical with the (5.5) plus $(5.6 \mathrm{~b})$ apart from the condition $j<l_{o m}$, which replaces $l_{m} \leq l_{o m}$.

\section{TIGHT TRANSITION STATE FOR ABC}

\section{A. General}

Equations (4.2) and (4.6) yield

$$
\sum_{j n l} w^{\kappa}=N_{\kappa \alpha}^{t},\left(|\kappa-j| \leq l \leq l_{<}, 0 \leq E_{j n} \leq E\right),
$$

where $l_{<}$is given by (4.12) and where $w^{\kappa}$ and $N_{k \alpha}^{t}$ denote

$$
\begin{aligned}
& w^{\kappa} \equiv w^{\kappa}\left(E_{\rho}-B_{l}^{\ddagger}\right), \\
& N_{\kappa \alpha}^{\ddagger} \equiv N_{\kappa \alpha}^{\ddagger}\left(E-B_{1 \kappa}^{\ddagger}\right),
\end{aligned}
$$

and $B_{i}^{\ddagger}$ and $E l$ are given by (4.7) and (4.8). A new quantity $B_{1 k}^{t}$ has been introduced: $B_{1 \kappa}^{\ddagger}$ is the energy of $A B C^{t}$ "fixed" as potential energy $U^{\ddagger}$ and as the part of the rotational energy associated with the total angular momentum quantum number $\kappa$, and so it cannot be distributed among the states $n^{\ddagger}$ of $A B C^{\ddagger}$. While $B_{l}^{\ddagger}$ can contribute only to $E_{p}$, during the formation of the products from $A B C^{\ddagger}, B_{1}^{\ddagger}$ can contribute both to the $E_{p}$ and $E_{j n}$ energies of the products.

When $\kappa \cong l$, the moment of inertia appearing in $B_{1 \kappa}^{t}$ [cf. Eq. (6.14) later] will be the same as that appearing in the exit channel barrier $B_{i}^{\ddagger}$, as in case (b) below. When $\kappa \cong j$, these two moments of inertia may differ, as in case (c) below.

The summation over $j, n, l$ is bounded by the limits in (6.1). $N_{\kappa \alpha}^{\ddagger}\left(E-B_{1 \kappa}^{\ddagger}\right)$ does not include a degeneracy factor $2 \kappa+1$, it will be recalled, which is absent from both sides. $N_{k \alpha}^{1}\left(E-B_{1 k}^{*}\right)$ is the number of active vibrationalrotational states of $\mathrm{ABC} C^{\ddagger}$, for given $E$ and $\kappa$, having an internal energy equal to or less than $E-B_{1 \kappa}^{\ddagger}$. Equation (6.1) is to be solved for the unknown function $w^{x}$.

\section{B. Tight transition state when $\ell_{0} \gg j_{0}$ and $\ell \gg>j$}

As in the corresponding case Sec. VB for the loose transition state the values of $l$ are clustered around the value of $\kappa$. The $l$ in $B_{l}^{l}$ in (6.2) is replaced by $\kappa$. Since 
$l \cong \kappa$, the moment of inertia in $B_{1 \kappa}^{*}$ is the same one as that in $B_{l}^{\ddagger}$, and so $B_{1 \kappa}^{\ddagger}$ in $(6.3)$ is replaced by $B_{\kappa}^{\ddagger}$.

The sum over $l$ in (6.1) is from $|\kappa-j|$ to $l_{<}$, where $l_{<}$ is, as in Sec. VB, set equal to $\kappa+j$ for almost all $k$ 's, because of the clustering of $l$ values. Since $|k-j|$ equals $k-j$, the sum over $l$ once again yields $2 j+1$. The sum of $2 j+1$ over $j$ and $n$, for $E_{p}$ in a range $\left(E_{p}\right.$, $\left.E_{p}+d E_{p}\right)$ yields by $(4.15) \rho\left(E-E_{p}\right) d E_{p}$, the number of rotation-vibration states for the products $\alpha$ in the range $E-E_{p}-d E_{p} \leq E_{j n} \leq E-E_{p}$. (It does not include the $2 \kappa+1$ degeneracy factor.)

Equation (6.1) then becomes

$$
\int_{E_{p}=B_{\kappa}^{\ddagger}}^{E} \rho\left(E-E_{p}\right) w^{\kappa}\left(E_{p}-B_{k}^{\ddagger}\right) d E_{p}=N_{\kappa \alpha}^{\ddagger}\left(E-B_{k}^{\ddagger}\right) .
$$

This equation is next solved for $w^{\kappa}$ by Laplace transforms: Multiplying both sides of (6.4) by $\exp [-s(E$ $\left.\left.-B_{k}^{\mathfrak{t}}\right)\right] d E$, integrating from $E=B_{k}^{\mathfrak{f}}$ to $\infty$, using the convolution theorem, and noting that the Laplace transform of the right-hand side of $(6.4)$ is $Q_{\kappa}^{\ddagger}(s) / s$, where $Q_{\kappa}^{\dagger}$ is defined below, we have

$$
\bar{w}^{\kappa}(s)=Q_{\kappa}^{\ddagger}(s) / s Q(s),
$$

where

$$
\begin{aligned}
& \bar{w}^{\kappa}(s)=\int_{0}^{\infty} w^{\kappa}(x) \exp (-s x) d x, \\
& Q^{\prime}(s)=\int_{0}^{\infty} \rho(x) \exp (-s x) d x, \\
& Q_{\kappa}^{\mathfrak{t}(s)}=\int_{0}^{\infty} \frac{d N_{k \alpha}^{\ddagger}(x)}{d x} \exp (-s x) d x .
\end{aligned}
$$

Inversion of (6.5) yields $w^{k}(x)$. Setting $x$ equal to $E_{p}$ $-B_{k}^{\dagger}$ we have

$$
\begin{aligned}
w_{j n l E}^{\kappa} & =w^{\kappa}\left(E_{p}-B_{1 \kappa}^{\ddagger}\right) \\
& =\frac{1}{2 \pi i} \int_{c-i \infty}^{c+i \infty} \frac{Q_{\kappa}^{\ddagger}(s)}{s Q(s)} \exp \left[s\left(E_{p}-B_{\kappa}^{\ddagger}\right)\right] d s,
\end{aligned}
$$

and with $l \cong \kappa$.

To obtain an expression for the cross section $\sigma_{j n ; j^{n} n_{0} E}$ using (4.13), it is first noted that since $\kappa \gg j$, the values of $l$ in the sum over $l$ are clustered around $l=\kappa$. Setting the $l$ in $w_{j n l E}^{\kappa}$ equal to some mean value $\kappa$ in this small interval of $l^{\prime} \mathrm{s}$, the sum over $l$ in (4.10) is from $\kappa-j$ to $\kappa+j$ and equals $(2 j+1) w_{j n t E}^{\kappa}$ with $l \cong \kappa$. We now have

$$
\left.\sigma_{j n ; j_{0} n_{0} E}=(2 j+1) \sigma_{0} \sum_{\kappa=0}^{\kappa_{<}} \frac{P_{0}(\kappa)}{\sum_{\alpha} N_{\kappa \alpha}^{t}} w_{j n l E}^{\kappa} \quad \text { (with } l \cong \kappa\right) \text {. }
$$

The upper limit $\kappa_{<}$in (6.10) is seen from (4.12) to be the smaller of $j+l_{m}$ and $\kappa_{m}$, i.e., of $l_{m}$ and $l_{o m}$ in this case of $l \gg j$ and $l_{0} \gg j_{0}$. Introducing Eq. (3.14) for $P_{0}(\kappa)$, Eq. (6.10) thus becomes

$$
\begin{aligned}
\sigma_{j n ; j 0 n_{0} E} & =(2 j+1) \frac{\sigma_{0}}{l_{o m}^{2}} \sum_{k=0}^{l_{o m}} \frac{2 \kappa+1}{\sum_{\alpha} N_{\kappa \alpha}^{t}} w_{j n l E}^{k} \text { for } l_{m}>l_{o m} \\
& =(2 j+1) \frac{\sigma_{0}}{l_{o m}^{2}} \sum_{k=0}^{l_{m}} \frac{2 \kappa+1}{\sum_{\alpha} N_{\kappa \alpha}^{t}} w_{j n l E}^{k} \text { for } l_{m}<l_{o m},
\end{aligned}
$$

where $w_{j n l B}^{\kappa}$ is given by (6.9).

In Eq. (6.11) the sum over $\kappa$ can be replaced by an integral over $2 \kappa d \kappa$, i. e., over $d\left(\kappa^{2}\right)$. Thus,

$$
\sigma_{j n ; j_{0} n_{0} E}=\frac{(2 j+1) \sigma_{0}}{\sum_{\alpha} N_{\kappa \alpha}^{t}} A_{t}\left(E_{p}\right),
$$

where the $t$ denotes "tight" and

$$
\begin{aligned}
A_{t}\left(E_{p}\right) & =\frac{1}{l_{o m}^{2}} \int_{\kappa^{2}=0}^{l_{o m}^{2}} w^{\kappa}\left(E_{p}-B_{\kappa}^{\mathfrak{t}}\right) d \kappa^{2} \quad\left(l_{m}>l_{o m}\right) \\
& =\frac{1}{l_{o m}^{2}} \int_{\kappa^{2}=0}^{l_{m}^{2}} w^{\kappa}\left(E_{p}-B_{\kappa}^{\ddagger}\right) d \kappa^{2} \quad\left(l_{m}<l_{o m}\right) .
\end{aligned}
$$

Here, the dependence of $\sum_{\alpha} N_{\kappa \alpha}^{t}$ on $\kappa$ has been presumed small, and the $\kappa$ in $N_{\kappa \alpha}^{\ddagger}$ represents some averaged value. (Otherwise, it can be placed in the integrand.)

From Eqs. (4.7) and (4.8), one sees that $B_{k}^{\ddagger}$ is the sum $U^{\ddagger}+\left[\kappa(\kappa+1) \hbar^{2} / 2 I^{\ddagger}\right] . I^{\ddagger}$, it will be recalled from Sec. IV, is usually taken to be a constant in the case of a tight transition state.

Equation (6.12) also applies to a loose transition state. For a loose transition state $Q_{k}^{\ddagger}$ equals $Q$ in (6.9), and so (6.9) reduces ${ }^{22}$ to the step function value for $w^{\kappa}$ given by (5.4), namely, unity if $E_{p}>B_{x}^{\ddagger}$ and zero if $E_{p}<B_{x}^{\ddagger}$. With this value for $w^{k} \mathrm{Eq}$. (6.12) yields (5.5).

\section{Tight transition state when $\ell_{0} \gg j_{0}$ and $j \gg \ell$}

In this case the $I^{t}$ associated with the centrifugal contribution in $B_{l}^{\ddagger}$ is expected to differ from the $I^{\ddagger}$ associated with the principal rotational quantum number of $\mathrm{ABC}^{\ddagger}, \kappa$. We shall denote the latter by $I_{1}^{\ddagger}$, so that $U^{\ddagger}$ plus the part of the rotational energy of $\mathrm{ABC}^{*}$ associated with $\kappa$ will be denoted by $B_{1 \kappa}^{\ddagger}$, where

$$
B_{1 \kappa}^{\ddagger}=U^{\ddagger}+E_{1 \kappa}^{\ddagger}=U^{\ddagger}+\kappa(\kappa+1) \hbar^{2} / 2 I_{1}^{\ddagger} .
$$

As before, we shall suppose that $E_{p}$ has to exceed a barrier $B_{l}^{\ddagger}$, where $B_{l}^{\ddagger}$ is given by (4.7).

In the sum over $j$ in Eq. (6.1), the $j$ 's are now clustered around the given value of $k$. In fact, $j$ ranges from $\kappa-l$ to $\kappa+l$, and $l \ll j$. Summing over $j$ in this cluster and neglecting the variation in $w^{\kappa}\left(E_{p}-B_{l}^{\dagger}\right)$ over this $j$ interval, the sum over $j$ equals $2 l+1$. The sum of $2 l+1$ over $l$ is from 0 to $l_{m}$. At any $j$ we may, in summing over $n$, introduce $\rho^{j}$, the density of the states defined by (4.16). Equation (6.1) becomes

$$
\int_{E_{p}=0}^{E} \rho^{j}\left(E-E_{p}\right) W^{\kappa}\left(E_{p}\right) d E_{p}=N_{\kappa \alpha}^{t}\left(E-B_{1_{\alpha}}^{t}\right),
$$

where $\kappa \cong j$ and where $W^{\kappa}$ is defined by

$$
W^{\kappa}\left(E_{p}\right)=\int_{l^{2}=0}^{t_{m}^{2}} w^{\times}\left(E_{p}-B_{l}^{\mathfrak{t}}\right) d l^{2}
$$

The integrand in (6.16) depends only on $E_{p}$ and $B_{l}^{\ddagger}$. The upper limit $l_{m}^{2}$ depends only on $E_{p}$ [cf. Eq. (4.9)]. Thus, the right-hand side of (6.16) depends only on $E_{p}$, a result explicitly indicated by the argument in $W^{k}\left(E_{p}\right)$.

Equation (6.15) is solved by a Laplace transformation. Both sides are multiplied by $\exp (-s E) d E$ and integrated from $E=0$ to $\infty$. The convolution theorem yields, as in (6.4) and (6.5),

$$
\bar{W}^{\kappa}(s)=\left[Q_{\kappa}^{\ddagger}(s) / s Q^{\kappa}(s)\right],
$$

where 


$$
\begin{aligned}
& \bar{W}^{\alpha}(s)=\int_{0}^{\infty} W^{\alpha}(y) \exp (-s) d y, \\
& Q_{\kappa}(s)=\int_{0}^{\infty}\left[d N_{\kappa \alpha}^{t}\left(y-B_{1 \kappa}^{\ddagger}\right) / d y\right] \exp (-s y) d y, \\
& Q_{j}(s)=\int_{0}^{\infty} \rho^{j}(y) \exp (-s y) d y .
\end{aligned}
$$

Inversion of (6.17) yields

$$
W^{\kappa}(y)=\frac{1}{2 \pi i} \int_{c-i \infty}^{c+i \infty} \frac{Q_{K}(s)}{s Q_{j}(s)} \exp s y d y .
$$

$Q_{x}^{\ddagger}(s)$ defined by (6.19) does not include the degeneracy $2 \kappa+1$, but the $y$ in (6.19) does include the energy $B_{1 k}^{t}$. The integrand in (6.19) does not become appreciable until $y$ exceeds $B_{1 \mathrm{k}}^{ \pm}$. The $\rho^{j}(y)$ in $(6.20)$ is also essentially zero until $y$, which is $E_{j n}$, has some minimal value $E_{j}$ for the rotational energy of the products consistent with the given $j$. When $E_{j}$ and the rotational energy in $B_{1 k}^{\ddagger}$ (with $\kappa \cong j$ ) are approximately equal, they tend to cancel in the ratio $Q_{\kappa}^{\ddagger}(s) / Q_{j}(s)$, and then $W^{\kappa}$ tends to be independent of or only weakly dependent on $j$.

The cross section is obtained from the reaction probability using Eq. (4.13). When $l_{o m}$, and hence $\kappa_{m}$, exceeds $j+l_{m}$, the $\kappa_{<}^{\prime}$ in (4.13) and (4.14) is $j+l$. Since $j>l, k$ varies from $j-l$ to $j+l$. If the variation of $w^{k}$ over this small $\kappa$ range is neglected, the sum of $(2 \kappa$ $+1) w^{\kappa}$ from $\kappa=j-l$ to $j+l$ is $(2 j+1)(2 l+1) w^{j}$. Thus, Eqs. (4.13) and (3.14) yield for this case of $l_{o m}>j+l_{m}$, i.e., of $l_{o m}>j$,

$$
\begin{aligned}
\sigma_{j n ; j 0 n_{0} E} & =\frac{(2 j+1) \sigma_{0}}{\left(\sum_{\alpha} N_{k \alpha}^{\ddagger}\right) l_{o m}^{2}} \int_{l^{2=0}}^{l_{m}^{2}} w^{j}\left(E_{p}-B_{l}^{\ddagger}\right) d l^{2} \\
& =\frac{(2 j+1) \sigma_{0}}{\sum_{\alpha} N_{k \alpha}^{\ddagger}} \frac{W^{j}\left(E_{p}\right)}{l_{o m}^{2}},\left(l_{o m}>j\right),
\end{aligned}
$$

where the $\kappa$ in $\sum_{\alpha} N_{k \alpha}^{t}$ is a suitably averaged value and $W^{j}\left(E_{p}\right)$ is given by $(6.21)$ with $\kappa \cong j$.

As in the loose case of Sec. VC, the cross section is zero when $l_{o m}$ and hence $\kappa_{m}$ is less than $j-l_{m}$.

From these results we have

$$
\sigma_{j n ; j_{0} n_{0} E^{E}}=\frac{(2 j+1) \sigma_{0}}{\sum_{\alpha} N_{\kappa \alpha}^{t}} A_{t}^{\prime}\left(E_{p}\right)
$$

where

$$
\begin{aligned}
A_{t}^{\prime}\left(E_{p}\right) & =0,\left(j>l_{o m}\right) \\
& =\frac{1}{2 \pi i l_{o m}^{2}} \int_{c-i \infty}^{c+i \infty} \frac{Q_{j}^{t}(s)}{s Q_{j}(s)}\left(\exp s E_{p}\right) d s,\left(j<l_{o m}\right) .
\end{aligned}
$$

As in Sec. VC, the conditions $l_{o m} \leqslant j-l_{m}$ and $l_{o m} \geqslant j+l_{m}$ were written as $l_{o m}<j$ and $l_{o m}>j$, since $l_{m} \ll j$. The $A_{t}^{\prime}\left(E_{p}\right)$ in (6.24) does not become appreciable until $E_{p}$ exceeds $B_{j}^{\prime}, E_{j}$.

Equation (6.23) reduces to Eq. (5.7) for the loose transition state when the assumptions appropriate to the latter are introduced.' For a loose transition state $Q_{j}^{\prime}$. can be written as

$$
Q_{j}^{t}(s)=Q_{f}(s) \int_{0}^{\infty} e^{-s B_{1}^{t}} 2 l d l
$$

Introduction of this result into the integral in (6.24) and integrating over $s$ first we have $e^{22}$

$$
\frac{1}{2 \pi i} \int_{c-i \infty}^{c+i \infty} \frac{Q_{j}^{\ddagger}(s)}{s Q_{j}(s)} \exp \left(s E_{p}\right) d s=\int_{0}^{\infty} 2 l d l h\left(E_{p}-B_{l}^{\ddagger}\right),
$$

where $h\left(E_{p}-B_{l}^{t}\right)$ is the unit step function, which is the same as the $w_{j n l E}^{k}$ in Eq. (5.4). Thereby, $h$ is unity if $l<l_{m}$ and zero if $l>l_{m}$ [cf. Eq. (4.9)]. The right-hand side of (6.26) thus equals $\int 2 l d l$ integrated from 0 to $l_{m}$, and so equals $l_{m}^{2}$. Thereby, Eq. (6.23) reduces to (5.7).

For a tight transition state the deviation of $Q_{f}^{f}(s) / Q_{f}(s)$ from the value in $(6.25)$ causes $A_{t}^{\prime}\left(E_{p}\right)$ to deviate from the value $(5.8)$ for the loose transition state.

\section{APPLICATIONS OF THE EQUATIONS IN SEC. VIB}

For purposes of illustration and further discussion, Eq. (6.9) for the tight transition state $l \gg j$ is evaluated for several examples. It will be recalled that the equations of Secs. VI B and VI C applied not only to a tight transition state for assumptions made, but also to a loose transition state, when the assumptions appropriate to the latter were introduced. Equations (5.5) and (5.7) were obtained.

Since $Q_{K}^{\ddagger} / Q$ in (6.9) was unity for a loose transition state, the deviation of $Q_{k}^{\ddagger}(s) / Q(s)$ from a value of unity in (6.9) represented the contribution of the steric effects to $w^{k}$ for the reverse step $\mathrm{AB}+\mathrm{C} \rightarrow \mathrm{ABC}$. (A loose transition state occurs for a reaction having no steric effects.) $Q(s)$ is known from the properties of $\mathrm{AB}+\mathrm{C}$. If $Q_{\kappa}^{\ddagger}(s)$ is calculated from the properties of the transition state and introduced into (6.9), the $w^{k}$ 's can be evaluated. The various cross sections can then be calculated from (6.12). With $l \gg j$, the $\kappa$ can be replaced by $l$ in the various equations.

For concreteness, two illustrations are given below for the case where $l \gg j$ and where the transition state is tight, i.e., for the case Sec. VIB.

\section{Example 1}

We first consider an example where the high frequency vibrations contribute little (close to a factor of unity) to $Q_{\kappa}^{t}(s)$ and to $Q(s)$ for the $s$ 's of interest. Their contribution to the ratio $Q_{k}^{\mathbb{t}}(s) / Q(s)$ is even closer to unity. We also replace, as previously noted, $\kappa$ by $l$ since $l \gg j$. If the contributions of the lower frequency motions to these partition functions are represented by

$$
Q_{\kappa}^{\ddagger}(s) \cong A^{\ddagger} / s^{m^{t}}, \quad Q(s) \cong A / s^{m},
$$

then

$$
Q_{\kappa}^{\ddagger}(s) / Q(s) \cong\left(A^{\ddagger} / A\right) s^{m-m^{\ddagger}} .
$$

Equation (6.9) is then readily evaluated to yield

$$
\begin{aligned}
w_{3 n l E}^{l} & \equiv w^{l}\left(E_{p}-B_{l}^{\ddagger}\right) \\
& =\frac{A^{\ddagger}}{A} \frac{\left(E_{p}-B_{l}^{\ddagger}\right)^{m^{\ddagger}-m}}{\Gamma\left(m^{\ddagger}-m+1\right)},\left(E_{p}>B_{l}^{\ddagger}\right) \\
& =0,\left(E_{p}<B_{l}^{\ddagger}\right),
\end{aligned}
$$


where $\Gamma(y)$ is the $\Gamma$ function of the argument $y$. When (7.1) is a sensible approximation, the right-hand side of (7.3) will not exceed unity, ${ }^{23}$ as indeed it should not. Equation (7.3) also reduces to Eq. (5.4) for a loose transition state, when the properties of the latter $\left(m^{t}\right.$ $=m, A^{\ddagger}=A$ ) are imposed.

When $\mathrm{AB}$ and $\mathrm{C}$ are a polyatomic molecule and an atom, respectively, two rotations of $\mathrm{AB}$ have become two bending vibrations of $\mathrm{ABC}^{*}$, in the reverse step of (1.2). When these bending vibrations are classical each contributes unity to $m^{\natural}$. Each rotation contributes $\frac{1}{2}$ to $m$. Thus, $m^{t}-m$ in this case equals unity.

When $\mathrm{AB}$ and $\mathrm{C}$ are both polyatomic molecules four rotations of $A B$ and of $C$ have become bending vibrations, yielding a classical contribution of 2 to $m^{\ddagger}-m$. Of the two remaining rotations one may become a vibration or restricted rotation in the transition states contributing perhaps $\frac{1}{2}$ to $m^{t}-m$. The sixth rotation remains as a rotation. Thus, in this case $m^{4}-m$ has a value of 22.5.

The value of $A_{t}\left(E_{p}\right)$ is calculated from (6.13) and (7.3). If one neglects any dependence of $I^{t}$ and $U^{\ddagger}$ on $l$, as one typically does in the case of a tight transition state but not in the case of a loose one, Eqs. (6.13) and (7.3) yield, ${ }^{24}$ noting that $\kappa \cong l$,

$$
\begin{aligned}
A_{t}\left(E_{p}\right)= & \frac{A^{\ddagger}}{A}\left(\frac{l_{m}^{2} \hbar^{2}}{2 I^{i}}\right)^{m^{\ddagger}-m}\left(\frac{l_{m}}{l_{o m}}\right)^{2} \\
& \times \frac{1-\left[1-\left(l_{o m} / l_{m}\right)^{2}\right]^{m^{\ddagger}-m+1}}{\Gamma\left(m^{\ddagger}-m+2\right)},\left(l_{m}>l_{o m}\right) \\
& =\frac{A^{\ddagger}}{A}\left(\frac{l_{m}^{2} \hbar^{2}}{2 I^{\ddagger}}\right)^{m^{\ddagger}-m}\left(\frac{\left(l_{m} / l_{o m}\right)^{2}}{\Gamma\left(m^{\ddagger}-m+2\right)}\right),\left(l_{m}<l_{o m}\right)
\end{aligned}
$$

Although (7.4) was explicitly derived for a tight transition state it also reduces to $A\left(E_{p}\right)$, given by (5.6), for a loose one. ${ }^{25}$

\section{Example 2}

For a second example we shall suppose that there is much cancellation of $Q_{k}^{t}$ and $Q$ in (6.9), apart from those contributions which are bending vibrations in $\mathrm{ABC}^{\ddagger}$ and rotations in $A B+C$. Let the number of these rotations be $m_{r}$. The rotations can be treated as classical. We let their contribution to $Q(s)$ be $Q_{r}(s)$, and write

$$
Q_{r}(s)=A_{r} / s^{m_{r} / 2},
$$

since each rotation contributes $\sqrt{s}$ to $s^{m_{r} / 2}$. The contribution of the bending vibrations to $Q_{k}^{\ddagger}(s)$ is denoted by $Q_{r}^{\ddagger}(s)$. Equation (6.9) now becomes, with $\kappa \cong l$,

$$
\begin{aligned}
w^{l}\left(E_{p}-B_{l}^{\ddagger}\right)= & \frac{1}{2 \pi i A_{r}} \int_{c-i \infty}^{c+i \infty} s^{\left(m_{r} / 2\right)-1} \\
& \times Q_{r}^{\ddagger}(s) \exp \left[s\left(E_{p}-B_{l}^{\ddagger}\right)\right] d s
\end{aligned}
$$

i. e.,

$$
=\frac{1}{A_{r}} \frac{d^{m_{r} / 2}}{d E_{p}^{m_{r} / 2}} N_{r}^{\mathfrak{t}}\left(E_{p}-B_{l}^{t}\right),
$$

Eq. (7.7) applying to the case that $m_{r}$ is an even integer. When $A B$ is a linear molecule and $C$ is an atom $m_{r}$ is 2. When $\mathrm{AB}$ and $\mathrm{C}$ are both polyatomic molecules and when the four of the six rotations become bending vibrations while the other two remain as rotations, $m_{r}$ is $4 . N_{r}^{\ddagger}$ can be evaluated by a direct counting technique as well as by more approximate methods. ${ }^{9,26}$ When $m_{r}$ is an odd integer (7.6) can be evaluated by a variety of methods. For example, $s^{-1 / 2} Q_{r}^{\mathbb{t}}(s)$ can be treated as a product of partition functions, the $s^{-1 / 2}$ being, apart from a proportionality constant, the partition function of a plane rotor. The corresponding "number of states" function will be denoted by $N_{r}^{\prime t}\left(E_{p}-B_{l}^{\ddagger}\right)$. The latter could be evaluated by direct counting or by approximate methods. Equation (7.6) then applies, with $N^{\ddagger}$ replaced by $N^{\prime t}$ and with $m_{r} / 2$ replaced by $m_{r}+\frac{1}{2}$, an even integer.

For the model in this example, $A_{t}\left(E_{p}\right)$ is obtained from $(6,13)$ and $(7.7)$. For the case where $m_{r} / 2$ is an integer we have, for a tight transition state,

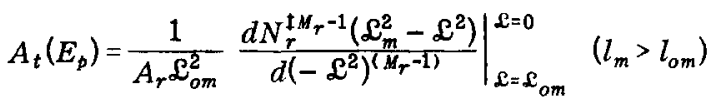

$$
\begin{aligned}
& =\left.\frac{1}{A_{r} \mathcal{L}_{o m}^{2}} \frac{d N_{r}^{1 H_{r}-1}\left(\mathscr{L}_{m}^{2}-\mathcal{L}^{2}\right)}{d\left(-\mathcal{L}^{2}\right)^{\left(H_{r}-1\right)}}\right|_{\mathscr{L}=\mathcal{L}_{m}} ^{\mathcal{L}=0} \quad\left(l_{m}<l_{o m}\right),
\end{aligned}
$$

where the right-hand side indicates a difference of the values derivative at the upper and lower limits, and where

$$
\mathcal{L}^{2}=l^{2} \hbar^{2} / 2 I^{\mathfrak{t}}, \quad M_{r}=m_{r} / 2 .
$$

$\mathscr{L}_{o m}$ is given by (7.9) with $l$ replaced by $l_{o m}$.

\section{TRANSLATIONAL ENERGY DISTRIBUTION OF REACTION PRODUCTS}

We shall consider the cases of Secs. VB, VC, VIB, and VIC in that order. In all cases Eq. (4.1) is used to relate $\sigma_{E_{p} ; j 0 n_{0} E}$ to $\sigma_{j n ; j 0^{n} 0} E$.

\section{(i) Case of Sec. VB (loose transition state, $\ell>>$ )}

Equation (5.5) is introduced into (4.1), noting that $A\left(E_{p}\right)$ does not depend on $j$ or $n$, for a given $E_{p}$. The sum $\sum_{j n}(2 j+1)$ over the energy range $E-E_{p}-d E_{p} \leqslant E_{j n}$ $-E_{p}$ is the number of vibrational-rotational states, $\rho\left(E-E_{p}\right) d E_{p}$, given by (4.15) for the $\alpha$ th set of products. Equations (4.1) and (5.5) then yield

$$
\sigma_{E_{p} ; j_{0} n_{0} E}=\frac{\sigma_{0}}{\sum_{\alpha} N_{k \alpha}^{t}} \rho\left(E-E_{p}\right) A\left(E_{p}\right),
$$

where $A\left(E_{p}\right)$ is given by (5.6). $l_{m}$ depends only on $E_{p}$; $l_{o m}$ depends only on $E_{p_{0}}$.

Equations (8.1) is equivalent to that derived by Safron et al. ${ }^{10,27}$ Thus, the latter equation yields the same result as phase space theory for the (highly useful) simplifying assumptions of $l \gg j$ and $l_{0} \gg j_{0}$. The latter assumptions were explicitly made in Ref. 10.

\section{(ii) Case of Sec. VC (loose transition state j $>\ell)$}

In Eq. (5.7), $A^{\prime}\left(E_{p}\right)$ depends only on $E_{p}$, for a given $E_{p_{0}}$ since $l_{m}$ in (5.8) depends only on $E_{p}$. Summing (5.7) over $j$ and $n$ in the energy range $\left(E-E_{p}-d E_{p} \leqslant E_{j_{n}} \leqslant E\right.$ $-E_{p}$ ) yields the sum $\sum_{j n}(2 j+1)$, which can again be written as $\rho\left(E-E_{p}\right) d E_{p}$. Equation (5.7) then yields 


$$
\sigma_{E_{p} ; j_{0}{ }^{n} 0^{E}}=\frac{\sigma_{0}}{\sum_{\alpha} N_{\mathrm{k} \alpha}^{\dagger}} \rho\left(E-E_{p}\right) A^{\prime}\left(E_{p}\right)
$$

where $A^{\prime}\left(E_{p}\right)$ is given by (5.8).

\section{(iii) Case of Sec. VIB (tight transition state, $\ell>>$ i)}

In Eq. (6.12), $A_{t}\left(E_{p}\right)$ depends only on $E_{p}$. On summing (6.12) over $j$ and $n$ in the energy range $E-E_{p}-d E_{p} \leqslant E_{j n}$ $\leqslant E-E_{p}$, one obtains $\sum_{j n}(2 j+1)$, i. e., the $\rho\left(E-E_{p}\right) d E_{p}$ in (4.15). Equations (4.1) and (6.12) yield

$$
\sigma_{E_{p} ; j_{0} n_{0} E}=\frac{\sigma_{Q}}{\sum_{\alpha} N_{k \alpha}^{t}} \rho\left(E-E_{p}\right) A_{t}\left(E_{p}\right),
$$

where $A_{t}\left(E_{p}\right)$ is given by $(6.13)$.

\section{(iv) Case of Sec. VIC (tight transition state $j>\ell$ )}

When the $j$ dependence of $Q_{j}^{\ddagger}(s) / Q_{j}(s)$ in (6.24) is weak, for the reason discussed in Sec. VI, the dependence of $A_{t}^{\prime}\left(E_{p}\right)$ on $j$ there is weak also. The summation of (6.23) over $j$ and $n$ in the energy interval $E-E_{p}-d E_{p}$ $\leqslant E_{j n} \leqslant E-E_{p}$ yields $\sum_{j n}(2 j+1)$, which is again the $\rho(E$ $\left.-E_{p}\right) d E_{p}$ in (4.15). Equations (4.1) and (6.23) then yield

$$
\sigma_{E_{p} ; j 0^{n} 0^{E}}=\frac{\sigma_{0}}{\sum_{\alpha} N_{k \alpha}^{t}} \rho\left(E-E_{p}\right) A_{t}^{\prime}\left(E_{p}\right),
$$

where $A_{t}^{\prime}\left(E_{p}\right)$ is given by (6.24).

\section{ROTATIONAL AND VIBRATIONAL ENERGY DISTRIBUTION OF REACTION PRODUCTS}

It is useful to first describe more fully in Eq. (9.1) below the quantum number $n$ appearing in Secs. IIIVIII. (Similar remarks apply to $n_{0}$. ) The collision pair in the reverse of step (1.2) is specified by the quantum numbers $j, n, l$ (and by the momentum $p$ ). The quantum numbers for a pair of molecules 1 and 2 in an uncoupled basis would be $j_{1}, m_{j_{1}}, k_{1}, j_{2}, m_{j_{2}}, k_{2}, l, m_{l}$, and $v$, where $v$ denotes the vibrational quantum numbers for all vibrational degrees of freedom of both molecules, $j_{1}$ and $j_{2}$ denote the rotational angular momentum quantum numbers of the molecules, $m_{j_{1}}$ and $m_{j_{2}}$ the $z$ components, and $k_{1}$ and $k_{2}$ are the extra rotational quantum numbers needed to specify the eigenvalues if the molecules are nonlinear. For example, $k_{1}$ is absent when molecule 1 is linear, and $j_{1}$ and $m_{1}$ are absent ${ }^{28}$ if molecule 1 is an atom. In a coupled basis, the quantum numbers are $j, l, \kappa, m_{\kappa}$, and $j_{1}, k_{1}, j_{2}, k_{2}, v$, the same number of them as before, of course. $m_{k}$ has already been included by the $2 \kappa+1$ factor present in $\mathrm{Eq}$. (3.9) for $P_{0}(\kappa)$. Thus, the quantum number $n$ denotes

$$
n \equiv\left\{j_{1}, k_{1}, j_{2}, k_{2}, v\right\},
$$

of which some of these may be absent if one of the product molecules is an atom or a linear molecule.

Thus, if the distribution of only certain of the quantum numbers of molecule $1, n_{1}$ say, is measured, the relevant cross section describing the distribution is

$$
\sigma_{n_{1} ; j_{0} n_{0} B}=\sum_{\substack{n \\\left(n \neq n_{1}\right)}} \sum_{j=\left|j_{1}-j_{2}\right|}^{j_{1}+j_{2}} \sigma_{j n ; j_{0} n_{0} B},
$$

where the notation is intended to indicate that the summation over $n$ is made at fixed $n_{1}$.

Regardless of whether 1 or 2 are linear or nonlinear, or whether 2 is an atom, the only dependence of $\sigma_{j n ; j_{0} n_{0} B}$ on $j$ lies in the $2 j+1$ factor in the case of cases of Secs. $\mathrm{VB}$ and VI B. In VC the only other dependence on $j$ is in the existence of a cutoff for $j>l_{o m}[\mathrm{Eq}$. (5.8)]. In Sec. VIC there is a similar cutoff in (6.24), and an additional weak $j$ dependence.

We consider cases of Secs. V B and VI B first. Here, the sum over $j$ in (9.2), using (5.5) or (6.12), involves a sum of $2 j+1$ from $j=\left|j_{1}-j_{2}\right|$ to $j_{1}+j_{2}$. This sum equals $\left(2 j_{1}+1\right)\left(2 j_{2}+1\right)$. In the subsequent sum in (9.2) over $n$ at fixed $n_{1}$ we note that $A\left(E_{p}\right)$ in Eq. (5.5) and $A_{t}\left(E_{p}\right)$ in Eq. (6.12) for $\sigma_{j n ; j_{0} n^{E}}$ do not depend on $n$ for a given $E_{p}$. The sum of $2 j_{2}+1$ over $j_{2}$ and the other contributors to $n$ is next written as the sum (integral) of contributions from various $d E_{p}$ intervals. The contribution to this $n$ sum from the $E_{j n}$ 's in the range indicated in (9.3), at the given $n_{1}$, is denoted by $\rho^{\left(n_{1}\right)}\left(E-E_{p}\right) d E_{p}$. That is,

$\sum_{\substack{n \\\left(n \neq n_{1}\right)}}\left(2 j_{1}+1\right)\left(2 j_{2}+1\right)=\rho^{\left(n_{1}\right)}\left(E-E_{n_{1}}-E_{p}\right) d E_{p}$,

$$
\left(E-E_{p}-d E_{p} \leqslant E_{j n} \leqslant E-E_{p}\right),
$$

where $E_{n_{1}}$ is the energy of the degrees of freedom contributing to the specified $n_{1}$ and $E-E_{n_{1}}-E_{p}$ is the energy distributed among the remaining rotations and vibrations of $\mathrm{AB}$ and $\mathrm{C}$.

Equations (9.2), (9.3), and (5.5) or (6.12) yield

$$
\sigma_{n_{1} ; j_{0} n_{0} E}=\frac{\sigma_{Q}}{\sum_{\alpha} N_{k \alpha}^{\ddagger}} \int_{E_{p}=0}^{E-E_{n_{1}}} \rho^{\left(n_{1}\right)}\left(E-E_{n_{1}}-E_{p}\right) C\left(E_{p}\right) d E_{p},
$$

where

$$
\begin{aligned}
C\left(E_{p}\right) & =A\left(E_{p}\right) \quad \text { (Sec. VB) } \\
& =A_{t}\left(E_{p}\right) \quad \text { (Sec. VIB), }
\end{aligned}
$$

and $A\left(E_{p}\right)$ and $A_{t}\left(E_{p}\right)$ are given by (5.6) and (6.13), respectively.

When $j_{1} \gg j_{2}$, so that the condition $j>l_{o m}$ or $<l_{o m}$ in (5.8) and (6.24) becomes $j_{1}>l_{o m}$ or $<l_{o m}$, and so does not depend on $j_{2}$, and when $j_{1}$ is one of the $n_{1}$ 's, (9.4) applies to the other cases (in the case of Sec. VIC if the cited weak $j$ dependence is neglected), but now

$$
\begin{aligned}
C\left(E_{p}\right) & =A^{\prime}\left(E_{p}\right) \quad(\text { Sec. V C }) \\
& =A_{t}^{\prime}\left(E_{p}\right) \quad \text { (Sec. VIC), }
\end{aligned}
$$

where $A^{\prime}$ and $A_{t}^{\prime}$ are given by (5.8) and (6.24), respectively, with $j$ replaced by $j_{1}$.

To illustrate (9.3) and (9.4), we note that if molecule 1 is a diatomic molecule and molecule 2 is an atom, and if $n_{1}$ denotes $j_{1}$ and $v_{1}$, the vibrational quantum number of molecule $1, \rho^{\left(n_{1}\right)}\left(E-E_{\phi}-E_{n_{1}}\right)$ equals $\left(2 j_{1}+1\right) \delta\left(E-E_{j_{1}}\right.$ $\left.-E_{v_{1}}-E_{p}\right)$ and Eq. (9.4) with $j_{1} \equiv j$ reduces to (5.5), $(5.7),(6.12)$, or $(6.23)$, depending on the case being studied.

If, instead, in this last example $n_{1}$ denotes only $j_{1}$, 
$\rho^{\left(n_{1}\right)}\left(E-E_{p}-E_{n_{1}}\right)$ equals $\left(2 j_{1}+1\right)$ times the density of vibrational states of molecule $1, \rho^{v} 1\left(E-E_{p}-E_{j_{1}}\right)$. Or again, if molecule 1 is a linear molecule and if molecule 2 is linear or nonlinear, and if $n_{1}$ denotes the rotationalvibrational state $\left(j_{1} v_{1}\right)$ of molecule $1, \rho^{\left(n_{1}\right)}\left(E-E_{p}-E_{n_{1}}\right)$ equals $\left(2 j_{1}+1\right)$ times the degeneracy, if any, associated with $v_{1}$, and times the density of rotational-vibrational states of molecule 2. If, in this last example, $n_{1}$ denotes only $j_{1}, \rho^{\left(n_{1}\right)}\left(E-E_{p}-E_{n_{1}}\right)$ is the convolution of the vibrational state density of molecule 1 and the rotational-vibrational state density of molecule 2 .

\section{CONCLUDING DISCUSSION}

Expressions for the various state-selected and energy distributions of products are given in Secs. V-IX. The distributions are seen to depend on the relevant degeneracies or densities of the active modes ${ }^{17}$ of the reaction products $\mathrm{AB}+\mathrm{C}$ and on the reaction probabilities for the reverse step in (1.2), $\mathrm{AB}+\mathrm{C}-\mathrm{ABC} \mathrm{B}^{*}$. This result is expected, because of the assumption regarding the role of active modes in $\mathrm{ABC}^{*}$ and because of microscopic reversibility. Assumption (iv) makes a particular assumption about these reaction probabilities, and, in conjunction with assumption (iii) yields an expression for them.

There is a considerable indirect body of data on the behavior of state-selected reaction probabilities for the case of direct reactions. However, little is known yet about those probabilities for reactions which may proceed via transient species, and there could be substantial differences. ${ }^{29}$

The expressions for loose and tight transition states have in common the presence of degeneracy or state density factors for the active modes, e.g., for the case of $l \gg j$, they have in Eqs. (8.1) and (8.3) $\rho\left(E-E_{p}\right)$ with $\rho\left(E-E_{\rho}\right)$ defined in (4.15). They differ in the reaction probabilities for the reverse step of (1.2), and so $A\left(E_{p}\right)$ in the former $[\mathrm{Eq} .(8.1)]$ is replaced by $A_{t}\left(E_{p}\right)$ in the latter [Eq. (8.3)]. Comparison of $A\left(E_{p}\right)$ with $A_{t}\left(E_{p}\right)$ shows that at higher $E_{p}$ 's (where $l_{m}>l_{o m}$ in exothermic reactions) $A_{t}\left(E_{p}\right)$ results in a predicted shift in $E_{p}$ distribution toward higher $E_{p}$ 's, compared with $A\left(E_{p}\right)$ [cf. (5.6a) with (6.13a)]. The origin of this predicted shift is discussed in Sec. I.

We have already noted that translational energy distribution of products of the loose transition state for the case of $l \gg j$ was treated earlier ${ }^{10}$ and that the present result [Eqs. (8.1) and (5.6)] agrees with that in Ref. 10. The case of a tight transition state was also discussed in Ref. 10 for the case of $l \gg j$, using a different model for the exit channel behavior. They assumed that the translational energy $E_{p}$ in the products of (1.2) was the same as that in $\mathrm{ABC}$, plus an amount $B_{l}^{\ddagger}$. In the present paper we assume that some conversion of bending vibrational to translational energy can occur, resulting in the tight transition state case (Sec. VIB) in $E_{p}$ being larger than the sum of the translational energy in $\mathrm{ABC}^{\ddagger}$ plus $B_{i}^{\dagger}$.

It is useful to compare the two results by considering an approximate model such as that involved in Sec. VII,
Example 1. However, we shall take all coordinates to be classical now, for purposes of the illustration. Each vibration in $\mathrm{ABC}^{1}$ (apart from the adiabatic one ${ }^{17}$ ) contributes 1 to the $m^{\ddagger}$ in the classical expression (7.1). Each active rotation in $\mathrm{ABC}^{\ddagger}$ contributes $\frac{1}{2}$. Thus, the value of $m^{t}$ is

$$
m^{t}=s^{t}+\frac{1}{2} r
$$

where $r$ is the number of "nonadiabatic" rotations in $\mathrm{ABC}^{\ddagger}$ and $s^{\ddagger}$ is the number of active vibrations in $\mathrm{ABC}^{\ddagger}$.30,31

The expression in Ref. 10 for the relative translational energy distribution is ${ }^{30}$

$$
\begin{aligned}
\sigma_{E_{p} ; j_{0} n_{0} E} & =\text { const. }\left(E-E_{p}\right)^{r / 2+s^{t}-1}, \quad l_{m} \geq l_{o m} \\
& =\text { const. }\left(l_{m} / l_{o m}\right)^{2}\left(E-E_{p}\right)^{r / 2+s^{\ddagger}-1}, l_{m}<l_{o m} .
\end{aligned}
$$

The dependence of $l_{m}$ on $E_{p}$ when a potential $-c / r^{n}$ is used for $U$ is given in Ref. 10.

For a loose transition state the density of states of the products $\rho\left(E-E_{\phi}\right)$ in $(8.1)$ is the same as that for the se coordinates in $\mathrm{ABC}^{\ddagger}$, namely, const. $\left(E-E_{p}\right)^{r / 2+s^{\ddagger}-1}$. Thus, Eq. (8.1) in this case is the same as Eq. (10.2), which was taken from Ref. 10.

For a tight transition state Eq. (8.3) gives

$$
\sigma_{E_{p} ; j 0 n 0 E}=\text { const. } A_{t}\left(E_{p}\right)\left(E-E_{p}\right)^{m-1}
$$

where

$$
m=r_{p} / 2+s_{p} \text {. }
$$

The $p$ subscript refers to products, $r_{p}$ being the number of active rotations of the products and $s_{p}$ the number of their active vibrations. [For a loose transition state $r_{p}=r$ and $s_{p}=s^{\ddagger}$.] In (10.3) $A_{t}\left(E_{p}\right)$ is given by (7.4). At high enough translational energies where $l_{o m} \ll l_{m}$, the latter equation for $A_{t}\left(E_{p}\right)$ yields

$$
A_{t}\left(E_{p}\right) \cong \text { const. } l_{m}^{2\left(m^{t}-m\right)},\left(l_{m} \gg l_{o m}\right) \text {. }
$$

For the model used in Ref. $10, l_{m}^{2}$ is roughly proportional to $E_{p}$. For this case one would then write

$$
\sigma_{E_{p} ; j 0 n_{0} E} \cong \text { const. } E_{p}^{m^{t}-m}\left(E-E_{p}\right)^{m-1},\left(l_{m} \gg l_{o m}\right) \text {, }
$$

which compares with (10.2a), i.e., with

$$
\sigma_{E_{p} ; j_{0} n_{0} E} \cong \text { const. }\left(E-E_{\phi}\right)^{m^{\ddagger}-1},\left(l_{m}>l_{o m}\right) \text {. }
$$

For a tight transition state, $m^{\ddagger}$ is larger than $m$ by an amount equal to one-half the number of bending vibrations which have become rotations. ${ }^{32}$ Thus, both because the factor $E_{p}^{\left(m^{-1}-m\right)}$ in $(10.6)$ increases with $E_{p}$ and because the factor $\left(E-E_{p}\right)^{m-1}$ decreases less rapidly with increasing $E_{p}$ than does $\left(E-E_{\phi}\right)^{m^{t}-1}$, the $E_{\phi}$ distribution predicted by $(10.6)$ is shifted toward higher $E_{p}$ 's, in the case of a tight transition state, than that predicted by the Eq. (10.7) based on Ref. 10. The physical explanation was given earlier in Sec. I.

\section{ACKNOWLEDGMENT}

I am indebted to Gary Worry for his comments. 
*Research supported by the Explosives Division, Feltman Research Laboratories, Picatinny Arsenal, Dover, N.J.

${ }^{1}$ See review by D. R. Herschbach, Faraday Discuss. Chem. Soc. 55, 233 (1973); cf. D. L. King and D. R. Herschbach, ibid. 55, 331 (1974); S. J. Riley and D. R. Herschbach, J. Chem. Phys. 58, 27 (1973).

${ }^{2}$ See the review by Y. T. Lee, Ber. Bunsenges Phys. Chem. 78, 135 (1974); Y. T. Lee, in Proceedings of the XI International Symposium on Free Radicals (Munich, West Germany, 1973); J. M. Farrar and Y. T. Lee, Ann. Rev. Phys. Chem. 25,357 (1974).

${ }^{3}$ J. G. Moehlmann, J. T. Gleaves, J. W. Hudgens, and J. D. McDonald, J. Chem. Phys. 60, 4790 (1974).

${ }^{4}$ P. J. Dagdigan, H. W. Cruse, A. Schultz, and R. N. Zare, J. Chem. Phys. 61, 4450 (1974).

${ }^{5}$ For example, R. A. Marcus and O. K. Rice, J. Phys. Colloid Chem. 55, 894 (1951), where the transition states were termed loose and rigid. Following the terminology in Ref. 1, the latter will now be called tight.

${ }^{6}$ P. Pechukas and J. C. Light, J. Chem. Phys, 42, 3281 (1965); P. Pechukas, J. C. Light, and C. Rankin, ibid. 44, 794 (1966).

${ }^{7}$ J. M. Parson, K. Shobatake, Y. T. Lee, and S. A. Rice, J. Chem. Phys. 59, 1402 (1972); K. Shobatake, J. M. Parson, Y. T. Lee, and S. A. Rice, ibid. 59, 1416 (1973); 59, 1427 (1973); K. Shobatake, Y. T. Lee and S. A. Rice, ibid. 59, 1435 (1973); J. M. Parson, K. Shobatake, Y. T. Lee, and S. A. Rice, Faraday Discuss. Chem. Soc. 55, 344 (1973); J. M. Parson and Y. T. Lee, J. Chem. Phys. 56, 4658 (1972). ${ }^{8}$ Compare Ref. 5; R. A. Marcus, J. Chem. Phys. 20, 359 (1952); 43, 2658 (1965); 52, 1018 (1970).

${ }^{9}$ P. J. Robinson and K. A. Holbrook, Unimolecular Reactions (Wiley, New York, 1972); W. Forst, Theory of Unimolecular Reactions (Academic, New York, 1973); L. D. Spicer and B. S. Rabinovitch, Ann. Rev. Phys. Chem. 21, 349 (1970).

${ }^{10}$ S. A. Safron, N. D. Weinstein, and D. R. Herschbach, Chem. Phys. Lett. 12, $564(1972)$; ef. W. B. Miller, S. A. Safron, and D. R. Herschbach, Disscuss. Faraday Soc. 44, 108 (1967).

${ }^{11}$ R. A. Marcus, J. Chem. Phys. 45, 2630 (1966); 46, 959 (1967).

${ }^{12}$ (a) The activation energy for halide + olefin is about $1 \mathrm{kcal} /$ mole [J. Knox and K, C. Waugh, Trans. Faraday Soc, 65, 1585 (1969); P. B. Ayscough, A. J. Cocker, F. S. Dainton, and S. Hirst, ibid. 58, 318 (1962)]. (b) The activation energy for $\mathrm{CH}_{3}+$ olefin is about $8 \mathrm{kcal} / \mathrm{mole}$ [cf. R. J. Cvetanovic and R. S. Irwin, J. Chem. Phys. 46, 1694 (1967); A. M. Hogg and P. Kebarle, J. Am. Chem. Soc. 86, 4558 (1964)] and that for $\mathrm{H}+$ olefin is about $3 \mathrm{kcal} / \mathrm{mole}$ [K. Yang, ibid. 84,3795 (1962); J. H. Knox and D. G. Dalgleish, Int. J. Chem. Kinet. 1, 69 (1969); W. E. Jones, S. D. Macknight, and L. Teng, Chem. Revs. 73, 407 (1973)]. None of the systems in (a) or (b) referred explicitly to the addition of these atoms or radical to fluoro-olefins. The presence of an $\mathrm{F}$ on a carbon atom to which the $\mathrm{H}$ is adding reduces the rate by a factor of $\sim 5-15$ [cf. data of J. P. Kilcoyne and K. R. Jennings, J. Chem. Soc. Faraday I 70, $379(1974)$; I am indebted to Professor B. S. Rabinovitch for referring me to this article]. Corresponding$\mathrm{ly}$, the activation energy could be $1-1.5 \mathrm{kcal} / \mathrm{mole}$ higher.

${ }^{13}$ R. A. Marcus, Faraday Discuss. Chem. Soc. 55, 379 (1974); 55,381 (1974); cf. discussion comments by several investigators on pp. $376-380$ of this journal.

${ }^{14}$ Phase space theory for (1.1) implies the assumption of a loose transition state for that step.

${ }^{15}$ For example, L. D. Landau and E. M. Lifschitz, Quantum Mechanics (Addison-Wesley, Reading, Mass., 1958), p. 437. Classically, it will be recalled that $(3.3)$ represents the contribution to $\sigma$ from the area element $2 \pi b d b$ times the reaction probability $w^{0}(b)$, from impact parameters in the range $(b, b+d b)$, and integrated from $b=0$ to $b=\infty$. Substitution of the semiclassical relation $\left(Z+\frac{2}{2}\right) \hbar=$ orbital angular momentum $=b p_{0}$, yields the integral counterpart of $(3.3), \pi \int_{0}^{\infty}(2 l+1) d l w^{0}(\mathrm{~b}) /$ $k_{0}^{2}$, where $k_{0}=p_{0} / \hbar$.

${ }^{16}$ Using the limits in $(3,13)$, the sum of $2 \kappa+1$ in the $P_{0}(\kappa)$ in (3.9) is from $\kappa=\left|j_{0}-l_{0}\right|$ to $j_{0}+l_{0}$ and equals $\left(2 l_{0}+1\right)\left(2 j_{0}+1\right)$. When $\left(\pi / k_{0}{ }^{2} \sigma_{0}\right)\left(2 l_{0}+1\right)$ is then summed over $l_{0}$ using the limits in (3.13) and using the $\sigma_{0}$ given by $(3.3)$ and $(3.4)$, the result is unity.

${ }^{17}$ The quantum number for an adiabatic degree of freedom remains constant during the process. Its energy may vary somewhat, since its energy depends not only on its quantum number but also on molecular properties such as its vibration frequency, anharmonicity, if any, etc. The energy of the adiabatic vibrations can be treated as being included in a potential energy function for the remaining coordinates. We shall suppose that this convenient device has been used. If all vibrations are active, i.e., if no vibrations are adiabatic, $s$ equals the number of vibrations of $A B C *$. For a sufficiently short-lived $A B C^{*}$ some of these vibrations may not be active.

${ }^{18}$ R. A. Marcus, J. Chem. Phys, 45, 2138 (1966).

${ }^{19}$ Ref. 18, Eq. (6), apart from a number of notational differences: The $n$ there includes the $n$ and $j$ here; $n \dagger$ denotes $n$; $J$ denotes $\kappa$; a transmission coefficient denoted there by $\kappa$ is set equal to unity here, since we are considering a classical case in the present paper. Adiabatic degrees of freedom there were denoted by $v$.

${ }^{20}$ The phase space theory result for three and four atom systems are given in Refs. 6 and 4, respectively, and summarized in Ref. 4. To relate Eqs. (3.9) plus (5.3) to Eqs. (A1) and (A3) of Ref. 4 the order of summation over $l_{0}$ and $\kappa$ in the former is interchanged, the new limits being given by (3.13).

${ }^{21}$ However, throughout, the primed and unprimed quantities in Ref. 10 refer to products and reactants, respectively, whereas we use unprimed and zero-subscripted quantities for the same purpose. Further, the subscript $t$ in Ref. 10 is the same as the present subscript $p$.

${ }^{22}$ A. Erdelyi, Tables of Integral Transforms (McGraw-Hill, New York, 1964), Vol, 1, p. 133.

${ }^{23} Q_{k} \ddagger / Q$ is unity for a loose transition state but less than unity for a tight one. One sees from (7.3) that the 1/s's of interest in (7.2) are of the order of $E_{p}-B_{l} \ddagger$. Thus, Eq. (7.2) and the condition that $Q_{x}^{\ddagger} / Q \leq 1$ implies that the right-hand side of (7.3) does not exceed unity, as long as (7.1) is a reasonable approximation.

${ }^{24}$ The fact that $E_{p}-U \hbar$ equals $l_{m}^{2} h^{2} / 2 I \ddagger$ was used.

${ }^{25}$ This paradox is explained by the fact that $(7.3)$ is valid for both loose and tight transition states, and the integral (6.13) is trivial for a loose transition state, so that the assumption that $I \ddagger$ and $U \ddagger$ in (7.4) are $l$ independent causes no error even when the transition state is loose.

${ }^{26}$ S. E. Stein and B. S, Rabinovitch, J. Chem. Phys, 58, 2438 (1973), and references cited therein.

${ }^{27}$ Compare with Eq. (1) of Ref. 10, where $A\left(E_{t}^{\prime}\right)$ is the same as $A\left(E_{p}\right)$. The $N_{v r}^{+}\left(E^{\prime}-E_{t}^{\prime}\right)$ is the same as our $\rho\left(E-E_{p}\right)$, since the internal degrees of freedom in a loose transition state are the same as those of the reaction products.

${ }^{28}$ Electronic angular momenta make a negligible contribution and have been ignored throughout. Any statistical factors to which they may contribute can be included at the appropriate places.

${ }^{29}$ In direct collisions, the potential energy surfaces of exothermic reactions are characterized by the terms "early downhill," "late downhill," as well as some intermediate category [cf. reviews by T. Carrington and J. C. Polanyi and by J. L. Kinsey, in MTP International Review of Science, Phys. Chem. Ser. I, Vol. IX. Chemical Kinetics, edited by J. C. Polanyi (Butterworths, London, 1972), Chaps. 5 and 6, respectively). The early downhill type refers to the case where the major decrease in potential energy along the reaction coordinate occurs before the region where the coordinate is highly curved in the usual skewed axis diagram. Late downhill refers to the case where the large decrease in potential energy occurs mainly after the system has passed through this 
curved region. The early downhill yields highly vibrationally excited reaction products, while the late downhill one causes translationally excited products. However, in the case of a three atom $A+B C \rightarrow A B+C$ reaction, this curved region is occupied by the $\mathrm{ABC}^{*}$. Thus, if an energy randomization occurs within an $\mathrm{ABC}^{*}$ molecule the above considerations do not apply. When an energy randomization does not cocur they do apply although in diluted form if there is some randomization. In either case, a knowledge of the nature of the potential energy surface from the $A B C^{*}$ region to the $A B+C$ region would be useful.

${ }^{30}$ This number is not to be confused with that of $A B C *$. When the transition state $A B C^{\ddagger}$ is tight, $s^{\ddagger}$ is one less than the number of active vibrations of $\mathrm{ABC}^{*}$, because one of the active vibrations in $\mathrm{ABC}^{*}$ has become the reaction coordinate in $\mathrm{ABC} \ddagger$. When the transition state is loose $s^{\ddagger}$ is more than one less than the number of active vibrations in $\mathrm{ABC}^{*}$, because some of those vibrations have become rotations.

${ }^{31}$ Cf. Eqs. (1), (8), and (12) of Ref. 10. The quantity denoted by $s-1$ in Ref. 10 is the same as our $s \neq$, since they include in their $s$ the reaction coordinate, whereas $s \ddagger$ does not. Other notational differences are noted in Ref. 27 of the present paper.

${ }^{32}$ The total number of degrees of freedom of $\mathrm{ABC} \ddagger$ in the center of mass system of coordinates is $r+s^{\sharp}+3$, since in addition to the $r+s$ there are two adiabatic rotations and one reaction coordinate. The total number of degrees of freedom of $\mathrm{AB}+\mathrm{C}$ in the center of mass system of coordinates is $r_{p}+s_{p}$ +3 , since in addition to the $r_{p}+s_{p}$ there are two orbital plus one radial degrees of freedom. Thus, $r+s \ddagger$ equals $r_{p}+s_{p}$. But $m \hbar-m$ is, by (10.1) and (10.4), therefore equal to $\left(s \ddagger-s_{p}\right) / 2$. 